\title{
Convenient Synthesis of Highly Functionalized, 3,4-Disubstituted Indole Building Blocks
}

\author{
Sonia Miranda, Pilar López-Alvarado, Carmen Avendaño and J. Carlos Menéndez*
}

Departmento de Química Orgánica y Farmacéutica, Facultad de Farmacia, Universidad Complutense, 28040 Madrid, Spain

\begin{abstract}
Several 3,4-disubstituted indole building blocks were synthesized, containing a one-carbon functional group at C-4 and a three-carbon chain bearing at least one $\omega$ functional group. A C-4 functionalized indole derivative was prepared by application of the Leimgruber-Batcho reaction. Several three-carbon chains were subsequently installed at the indole C-3 position. In the first strategy employed, a Mannich reaction was followed by the creation of a C-C bond by phosphine-induced generation of a 3-methyleneindolenine species, which was trapped by diethyl malonate. Alternatively, a Vilsmeier formylation at C-3 was followed by Knoevenagel or Wittig reactions or by treatment with ethyl diazoacetate in the presence of a Lewis acid. Direct introduction of the three-carbon chain at C-3 using a Lewis acid-catalyzed Michael reaction was also achieved. Further functionalization of the benzylic position attached to $\mathrm{C}-4$ by attachment of a nitro group was carried out, but preparation of a tricyclic welwistatin fragment by intramolecular nitroalkane acylation or intramolecular Henry reactions was not possible.
\end{abstract}

\section{INTRODUCTION}

Many bioactive natural and unnatural products contain 3,4-disubstituted indole or oxindole frameworks. The structures of a few representative examples are shown in Fig. 1, including ergotamine-type alkaloids such as lysergic acid [1], the antibiotic spearadine [2] and the MDR inhibitor and antimicrotubule agent welwistatin [3], a product of the metabolism of the cyanobacteria Wetsiella intricata and Hapalosiphon welwtschii [4].<smiles>CN1C[C@H](C(=O)O)C=C2Cc3cccc4[nH]cc(c34)[C@H]21</smiles>

Lysergic acid

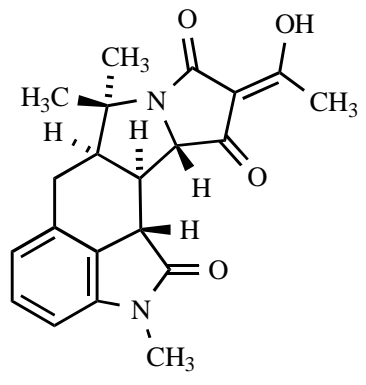

Speradine A

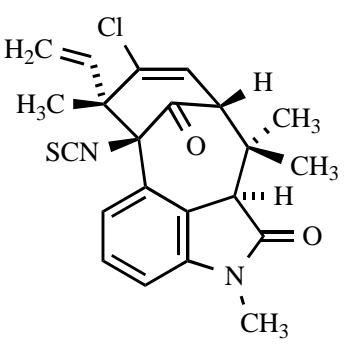

Welwistatin
Fig. (1). Structures of some bioactive alkaloids containing a 3,4disubstituted indole or oxindole fragment.

*Address correspondence to this author at the Departmento de Química Orgánica y Farmacéutica, Facultad de Farmacia, Universidad Complutense, 28040 Madrid, Spain; E-mail: josecm@farm.ucm.es
A large number of methods are known that allow the synthesis of substituted indoles [5,6,7]. Nevertheless, the preparation of 3,4-disubstituted indole systems is still a very challenging task, and the difficulties found in this endeavour can be partly attributed to the low nucleophilicity of the indole C-4 position. Although some methodologies involving the use of transition metal complexes or anionic benzyne cyclizations have been created for this purpose [8], the development of efficient methods leading to 3,4-disubstituted indoles from simple starting materials continues to be of great interest. We describe in this paper our route to several highly functionalized building blocks containing this structural motif.

\section{RESULTS AND DISCUSSION}

The starting material that we chose for our study was ethyl indole-4-carboxylate, which was obtained using the Leimgruber-Batcho indole synthesis [9], based on the treatment of 2-nitrotoluene derivatives with dimethylformamide acetals. As shown in Fig. 2, treatment of commercially available methyl-2-methyl-3- nitrobenzoate with dimethylformamide diethylacetal gave a $95 \%$ yield of compound $\mathbf{1}$, where enamine formation was accompanied by transesterification by the ethanol liberated from the DMF acetal. Its reductive cyclization in the presence of Ni Raney afforded indole 2a, which was $N$-methylated to $\mathbf{2 b}$ by treatment with sodium hydride and methyl iodide. Compounds $\mathbf{2 a}$ and $\mathbf{2 b}$ were efficiently reduced by exposure to lithium aluminium hydride to give alcohols 3a and $\mathbf{3 b}$, respectively, which were finally protected as tert-butyldiphenylsilyl ethers $\mathbf{4 a}$ and $\mathbf{4 b}$.

The Mannich reaction allows the easy creation of a carbon-carbon bond at the indole C-3 position, and the dimethylamino group can be subsequently displaced to give highly electrophilic 3-methyleneindolenine species. For this reason we treated an acetic acid solution of compounds $\mathbf{3 a}, \mathbf{b}$ with formaldehyde and dimethylamine, yielding Mannich bases 5a,b. In order to facilitate displacement of the dimethylamino group we methylated compounds $\mathbf{5}$ to give gramine 
derivatives 6a,b, whose reactivity towards the diethyl malonate anion was subsequently studied. Although we hoped that use of an excess of anion would allow the use of hydroxyl-unprotected starting materials, the reaction of the sodium derivative of diethyl malonate with $\mathbf{6 a}$ proved to be not possible. On the other hand, we achieved limited success ( $22 \%$ yield of compound $7 \mathbf{b}$ ) by treatment of $\mathbf{6 b}$ with an ethanolic solution of diethyl malonate in the presence of sodium ethoxide. Numerous attempts to improve this result by changing the base $(\mathrm{NaH})$, the solvent (DMF) and the reaction conditions (time, temperature, excess of malonate anion) were unsuccessful. In view of these problems, compound 5a was transformed into its $O$-silyl protected derivative $\mathbf{8}$, which was treated with diethyl malonate in the presence of tributylphosphine as a catalyst [10], giving compound $\mathbf{9}$ in an acceptable $48 \%$ yield (Fig. 3 ).<smiles>CCCCCN(C)C(OC)OC</smiles><smiles>CCOC(=O)c1cccc([N+](=O)[O-])c1/C=C/N(C)CC1CC1</smiles>

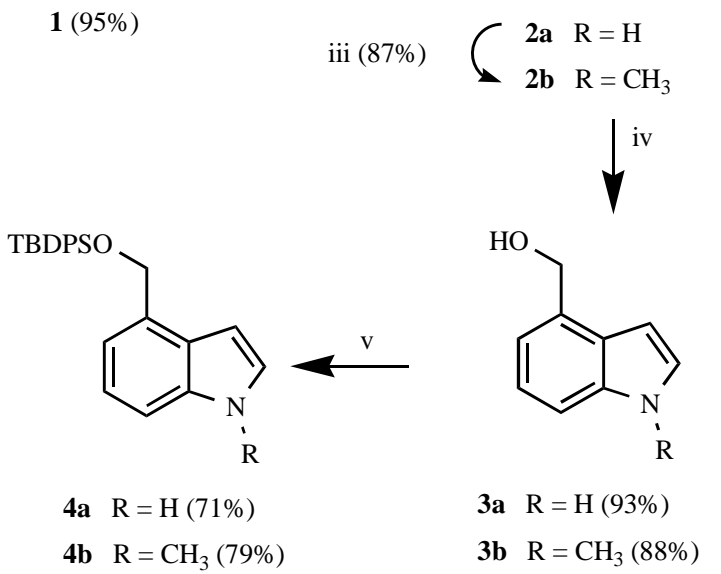

Reagents and conditions: i. DMF, $110{ }^{\circ} \mathrm{C}, 48$ h, ii. $\mathrm{NH}_{2}-\mathrm{NH}_{2} \mathrm{H}_{2} \mathrm{O}$, Ni Raney, $\mathrm{CH}_{3} \mathrm{OH}-\mathrm{THF}$, r.t., $45 \mathrm{~min}$. iii. $\mathrm{NaH}$, DMF, r.t., $5 \mathrm{~min}$, then $\mathrm{ICH}_{3}$, DMF, r.t., 1 h: iv. $\mathrm{LiAlH}_{4}$, THF, r.t., $12 \mathrm{~h}$ for $3 \mathrm{a}, 48 \mathrm{~h}$ for $3 \mathrm{~b}$; v. TBDPSCl, $\mathrm{Et}_{3} \mathrm{~N}$, DMAP, $\mathrm{Cl}_{2} \mathrm{CH}_{2}$, r.t., 12 h.

Fig. (2). Synthesis of 4-substituted indoles as starting materials.

Another approach to the functionalization of the C-3 position involved the use of the Vilsmeier-Haack formylation. Treatment of compound $\mathbf{4 b}$ with phosphorous oxychloride in dimethylformamide afforded an iminium salt $\mathbf{1 0}$ that could be isolated or hydrolyzed in situ under basic conditions $(\mathrm{pH}$ $8,60^{\circ} \mathrm{C}$ ) to yield aldehyde 11 , while the use of harsher basic

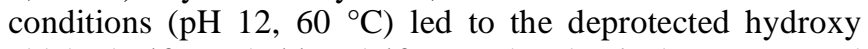
aldehyde 12. Both 11 and 12 gave the classical Knoevenagel reaction with diethyl malonate, albeit in moderate yields, affording compounds $\mathbf{1 3}$ and $\mathbf{1 4}$, respectively (Fig. 4).

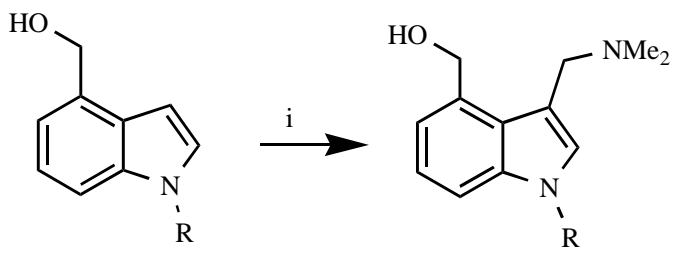
3a $\mathrm{R}=\mathrm{H}$
5a $\mathrm{R}=\mathrm{H}(92 \%)$
3b $\quad \mathrm{R}=\mathrm{CH}_{3}$
5b $\quad \mathrm{R}=\mathrm{CH}_{3}(86 \%)$

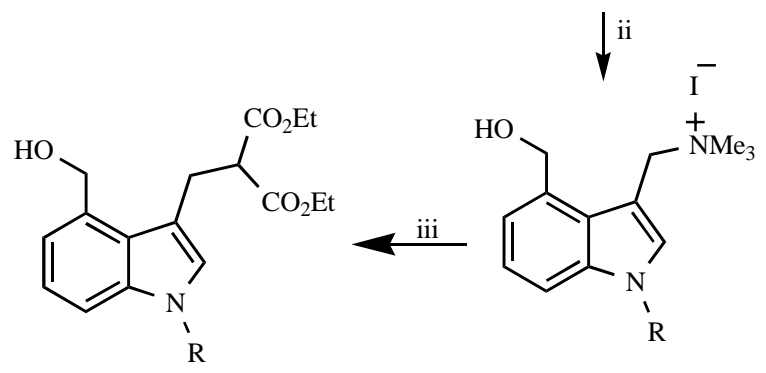

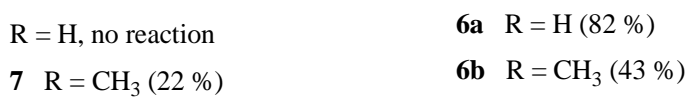

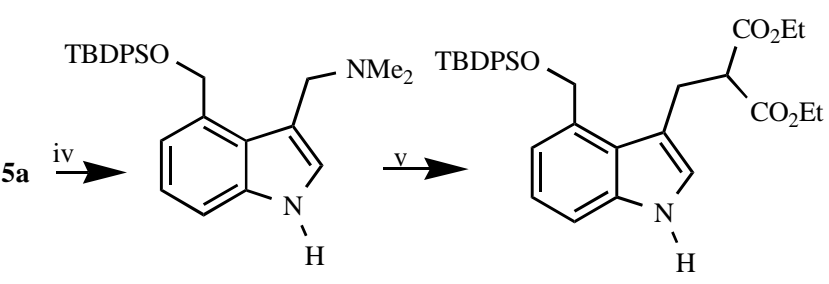

$8(66 \%)$

$9(48 \%)$

Reagents and conditions: i. $\left(\mathrm{CH}_{3}\right)_{2} \mathrm{NH}, \mathrm{HCHO}, \mathrm{H}_{2} \mathrm{O}, \mathrm{AcOH}$, r.t., 3 h. ii. $\mathrm{ICH}_{3}, \mathrm{THF}, 4^{\circ} \mathrm{C}, 12 \mathrm{~h}$. iii. NaOEt, diethyl malonate, $\mathrm{EtOH}$, reflux, $12 \mathrm{~h}$; iv. TBDPSCl, $\mathrm{Et}_{3} \mathrm{~N}$, DMAP, $\mathrm{Cl}_{2} \mathrm{CH}_{2}$, r.t., 12 h; v. Diethyl malonate, $\mathrm{PBu}_{3}$, THF, $80{ }^{\circ} \mathrm{C}, 24 \mathrm{~h}$

Fig. (3). Synthesis of 3,4-disubstituted indoles based on the Mannich reaction of compounds 3 .

In search for an alternative, more efficient procedure to create a three-carbon chain at $\mathrm{C}-3$, we carried out the reaction of compound $\mathbf{1 1}$ with ethyl diazoacetate in the presence of a Lewis acid [11] and obtained compound $\mathbf{1 5}$ in moderate yield. The most efficient method for the installation of the three-carbon chain proved to be the Wittig reaction of aldehydes 11 or 12 with ethoxycarbonylmethylene-triphenylphosphorane, which afforded good to excellent yields of compounds 16 and $\mathbf{1 7}$, respectively. Interestingly, the Wittig reaction leading to $\mathbf{1 6}$ could also be carried out from iminium salt 10, although a longer reaction time was required. Catalytic hydrogenation of $\mathbf{1 6}$ and $\mathbf{1 7}$ furnished $\mathbf{1 8}$ and $\mathbf{1 9}$, respectively, in quantitative yields, and compound 19 could also be obtained very efficiently by fluoride-induced deprotection of $\mathbf{1 8}$ (Fig. 5).

In order to allow the generation of a nucleophilic center at the benzylic position attached to C-4, we planned the introduction of a nitro group by displacement of a suitable halide with sodium nitrite. This transformation proved troublesome, since the application of published procedures for the transformation of halides into nitroalkanes $[12,13]$ to the 


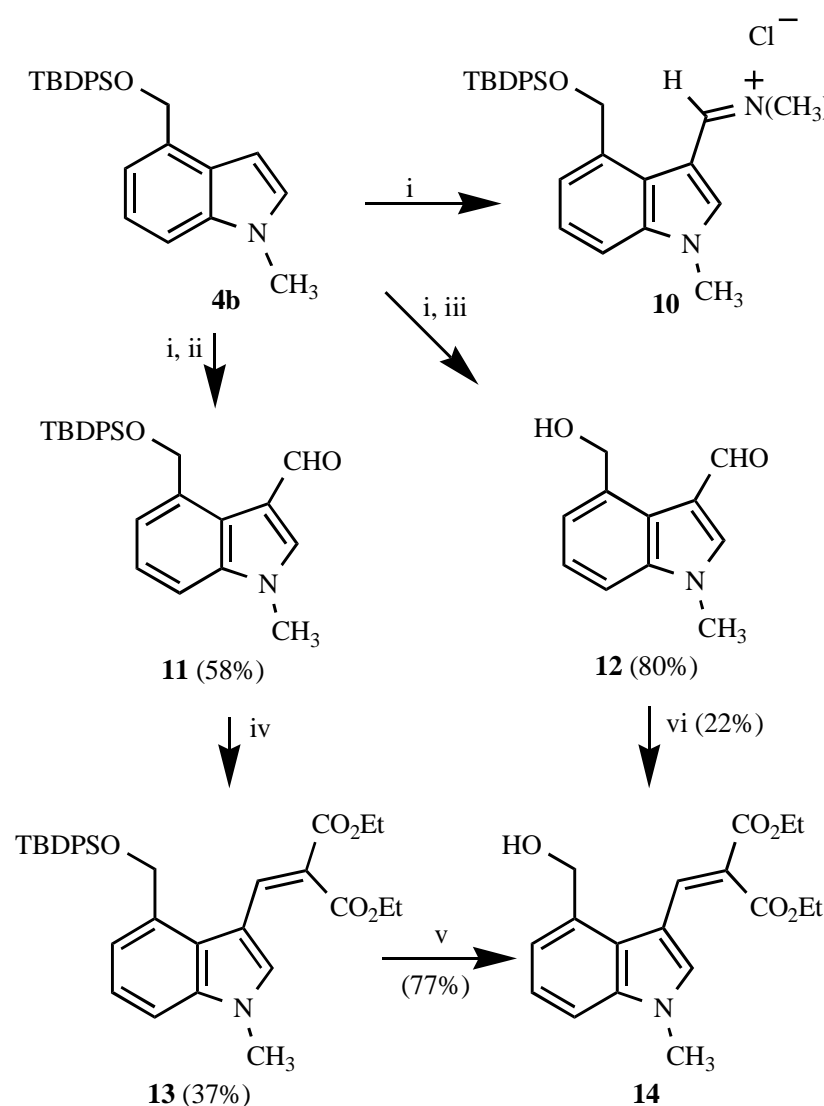

Reagents and conditions: i. $\mathrm{POCl}_{3}$, DMF, $0{ }^{\circ} \mathrm{C}, 30 \mathrm{~min}, 0{ }^{\circ} \mathrm{C}$ to r.t., 45 min. ii. $\mathrm{NaOH}-\mathrm{H}_{2} \mathrm{O}, \mathrm{pH}=8,60{ }^{\circ} \mathrm{C}, 30 \mathrm{~min}$; iii. $\mathrm{NaOH}-\mathrm{H}_{2} \mathrm{O}, \mathrm{pH}=12,60$ ${ }^{\circ} \mathrm{C}, 30 \mathrm{~min}$; iv. Diethyl malonate, pyridine-piperidine, $180{ }^{\circ} \mathrm{C}, 40 \mathrm{~h}$. v. Diethyl malonate, pyridine-piperidine, $180{ }^{\circ} \mathrm{C}, 4$ days; vi. $\mathrm{HF}, \mathrm{CH}_{3} \mathrm{CN}$, r.t., $24 \mathrm{~h}$

Fig. (4). Initial attempts at the installation of a three-carbon chain at the indole C-3 position using the Knoevenagel reaction.

reaction between sodium nitrite and benzyl bromide, used as a model, did not lead to the expected phenylnitromethane but to benzyl nitrite as the only product. This problem could be overcome by addition of urea to the reaction mixture in order to increase the solubility of sodium nitrite in the dimethylformamide used as solvent [14], and under the new conditions $\left(-20^{\circ} \mathrm{C}\right.$, urea, DMF) the model reaction gave phenylnitromethane in $80 \%$ yield. This result was further improved by the use of benzyl iodide as the substrate, which led to the desired nitro derivative in $99 \%$ yield after $5 \mathrm{~h}$ at $-20^{\circ} \mathrm{C}$. For this reason, alcohol 19 was transformed into iodide $\mathbf{2 0}$ using the one-pot protocol developed by Olah, involving treatment with trimethylsilyl chloride and potassium iodide $[15,16]$. The reaction of iodide 20 with sodium nitrite was carried out under similar conditions, although at a lower reaction temperature, and gave nitro derivative $\mathbf{2 1}$ in 54\% yield, together with a small amount of alcohol 19, presumably from hydrolysis of the iodide. Unfortunately, all attempts to induce a 7-exo-trig cyclization from the anion of 21, prepared using several bases including DBU in acetonitrile [17], KFalumina suspended in THF [18] and BuLi in THF-DMU [19], were unsuccessful. An attempt to facilitate the cyclization by activating the ester group in $\mathbf{2 1}$ via its transformation into the corresponding Weinreb amide [20] was also unsuccessful (Fig. 6).<smiles>[R20]Cc1cccc2c1c(C=O)cn2C</smiles>

$$
11 \mathrm{R}=\text { TBDPSO } 15(25 \%)
$$

$12 \mathrm{R}=\mathrm{H}$<smiles>[R20]Cc1cccc2c1c(CCC(=O)OCC)cn2C</smiles>

$$
\left.\begin{array}{lc}
\mathbf{1 6} \mathrm{R}=\mathrm{TBDPSO} & \mathbf{1 8} \mathrm{R}=\mathrm{TBDPSO} \\
(E / Z=9: 1,87 \%) & \mathbf{1 0 0 \% )} \mathrm{R}=\mathrm{H}(95 \%)
\end{array}\right) \text { iv }(95 \%)
$$

Reagents and conditions: $\mathrm{i} . \mathrm{N}_{2} \mathrm{CHCO}_{2} \mathrm{Et}, \mathrm{BF}_{3}-\mathrm{Et}_{2} \mathrm{O}, \mathrm{CH}_{2} \mathrm{Cl}_{2}$, r.t., 4 h; ii. $\mathrm{Ph}_{3} \mathrm{PCHCO}_{2}$ Et, EtOH, reflux, 48 h; iii. $\mathrm{H}_{2}, \mathrm{Pd}-\mathrm{C}, \mathrm{CH}_{3} \mathrm{OH}, 9$ h; iv. TBAF, THF, r.t., $10 \mathrm{~h}$.

Fig. (5). Installation of a three-carbon chain at the indole C-3 position using diazoalkane and phosphorous ylide chemistry.<smiles>CCOC(=O)CCc1cn(C)c2cccc(CO)c12</smiles>

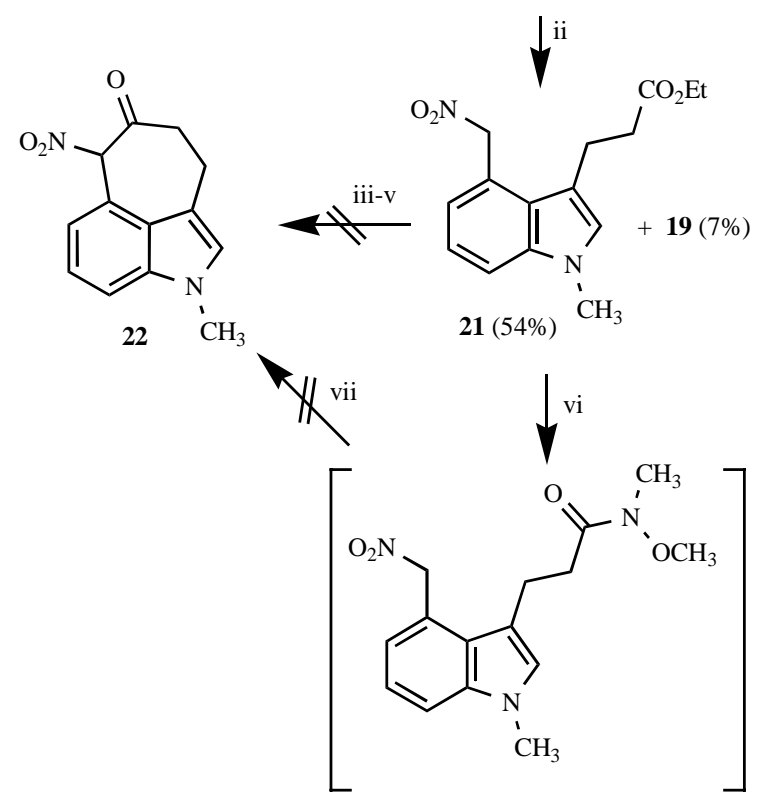

Reagents and conditions: i. $\mathrm{NaI}$ (2 eq.), $\mathrm{TMSCl}\left(2\right.$ eq.), $\mathrm{CH}_{3} \mathrm{CN},-75$ to $40{ }^{\circ} \mathrm{C}, 2 \mathrm{~h}$. ii. $\mathrm{NaNO}_{2}$, urea, DMF, $-75^{\circ} \mathrm{C}$ to $-40{ }^{\circ} \mathrm{C}, 2 \mathrm{~h}$; iii. DBU, $\mathrm{CH}_{3} \mathrm{CN}$, several conditions; iv. KF-alumina, THF, several conditions; v. BuLi, THF, DMU, several conditions; vi. $\left(\mathrm{CH}_{3}\right)_{2} \mathrm{AlCl}, \mathrm{H}_{3} \mathrm{CONHCH}_{3} \mathrm{HCl}$, $\mathrm{CH}_{2} \mathrm{Cl}_{2}$, r.t., $4 \mathrm{~h}$; vii. DBU, THF, several conditions.

Fig. (6). Further functionalization of compound 19 and attempts at the preparation of a welwistatin tricyclic fragment. 
In an effort to achieve the desired cyclization by an intramolecular Henry reaction, ester $\mathbf{1 8}$ was treated with lithium aluminium hydride to give primary alcohol $\mathbf{2 3}$, which was then oxidized to aldehyde $\mathbf{2 4}$ by exposure to the LeyGriffith TPAP reagent [21]. Since an attempt at the deprotection of the hydroxyl group in $\mathbf{2 4}$ led to a complex mixture of products, a decision was made to protect its aldehyde group as a dimethyl acetal, which was prepared using the mild conditions described by Noyori [22] in order to avoid decomposition of the indole ring. Acetal 25 thus prepared could be deprotected in the presence of fluoride to give compound 26, but all attempts to transform this compound into the corresponding iodide using Olah's conditions led only to complex mixtures of decomposition products. The intermediate of this reaction, i.e., trimethylsilyl ether $\mathbf{2 7}$, was isolated by carrying out the procedure in the presence of triethylamine (Fig. 7), but again this compound could not be transformed into the desired iodide.

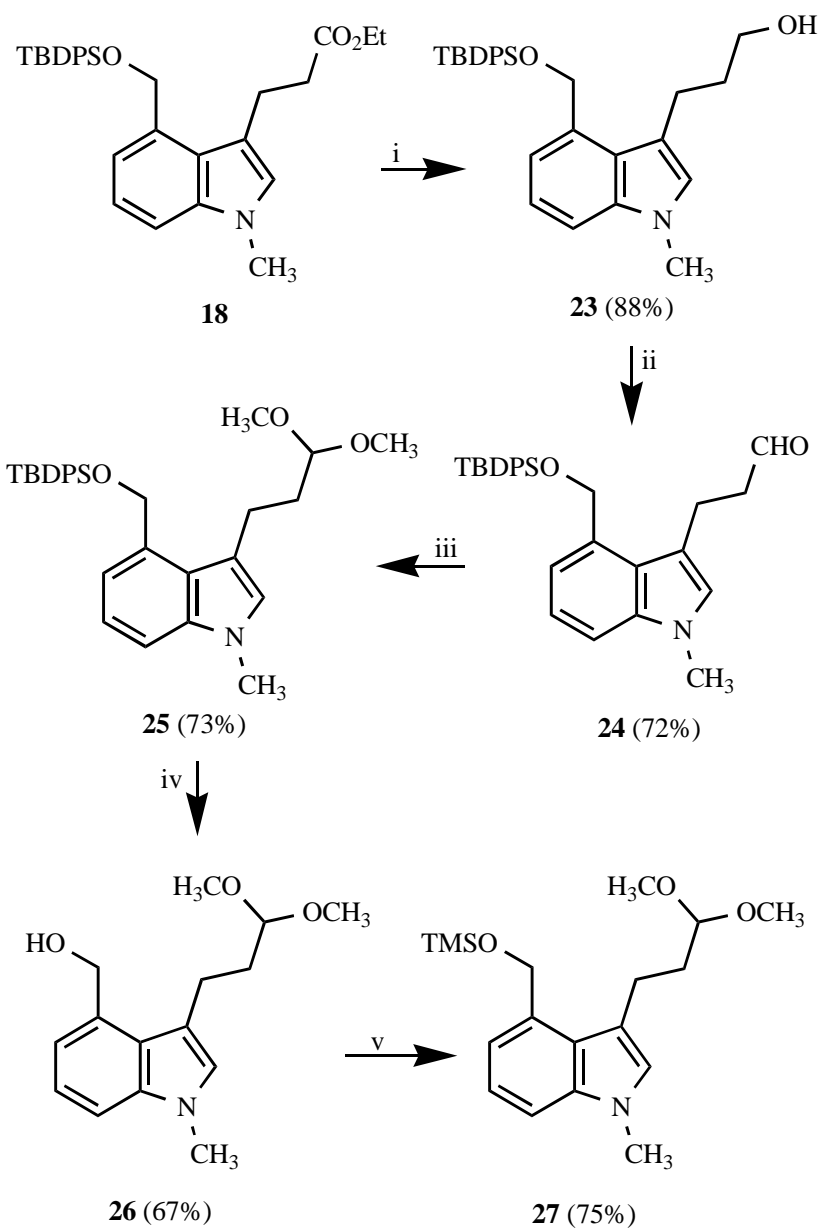

Reagents and conditions: i. $\mathrm{LiAlH}_{4}$, THF, r.t., 16 h; ii. TPAP, NMO, $\mathrm{CH}_{3} \mathrm{CN}$, r.t., $3 \mathrm{~h}$; iii. TMSOTf, methoxytrimethylsilane, $\mathrm{CH}_{2} \mathrm{Cl}_{2},-78$ ${ }^{\circ} \mathrm{C}, 3 \mathrm{~h}$; iv. $\mathrm{Bu}_{4} \mathrm{~N}^{+} \mathrm{F}^{-}$, THF, r.t., 32 ; v. NaI, TMSCl-Et $\mathrm{N}_{3} \mathrm{~N},-78{ }^{\circ} \mathrm{C}$ to $40{ }^{\circ} \mathrm{C}, 90 \mathrm{~min}$.

Fig. (7). Functionalization of compound 18.

In order to avoid the use of carbonyl-protecting groups, an alternative approach involving the use of a ketone group in the side chain was conceived. As shown in Fig. 8, the ytterbium triflate-catalyzed [23] Michael reaction of $\mathbf{4 b}$ with methyl vinyl ketone afforded compound $\mathbf{2 8}$, which in this case could be deprotected to $\mathbf{2 9}$ by treatment with fluoride. Exposure of 29 to the previously employed Olah's conditions allowed the preparation of iodide $\mathbf{3 0}$, which furnished nitro derivative $\mathbf{3 1}$ in moderate yield, together with alcohol 29, upon treatment with sodium nitrite and urea in dimethylformamide. Compound $\mathbf{3 1}$ was then submitted to literature conditions that allow to carry out the Henry reaction between nitroalkanes and ketones by displacing the unfavourable equilibria through reaction of the nitroaldol adduct with a thiol [24]. Unfortunately, all our attempts at translating these conditions into an intramolecular reaction allowing the preparation of compound $\mathbf{3 2}$ from $\mathbf{3 1}$ were unsuccessful.

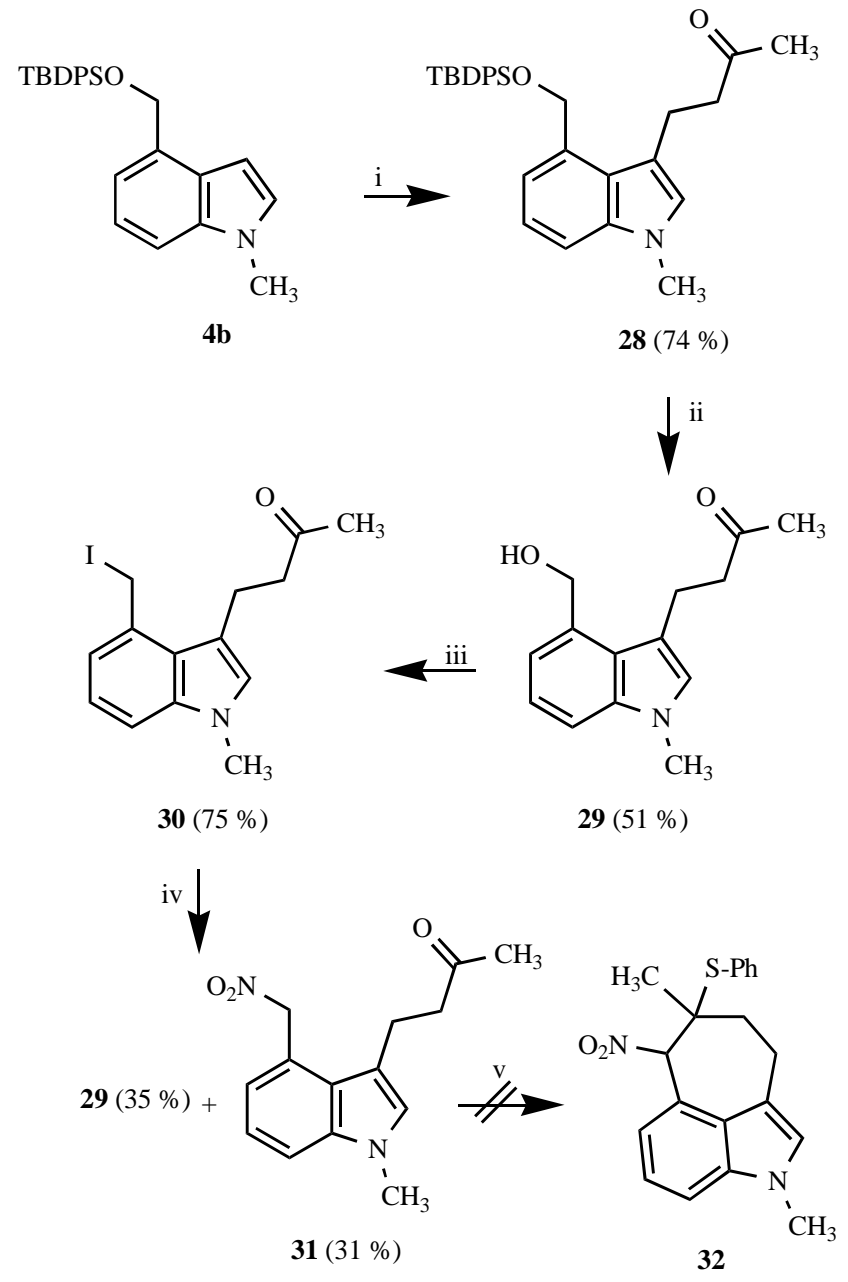

Reagents and conditions: $\mathrm{i}$. Methyl vinyl ketone, $\mathrm{Yb}(\mathrm{OTf})_{3} .3 \mathrm{H}_{2} \mathrm{O}$, r.t., 16 h; ii. $\mathrm{Bu}_{4} \mathrm{~N}^{+} \mathrm{F}^{-}$, THF, r.t., $12 \mathrm{~h}$; iii. $\mathrm{NaI}, \mathrm{TMSCl}, \mathrm{CH}_{3} \mathrm{CN},-78$ ${ }^{\circ} \mathrm{C}$ to $-40{ }^{\circ} \mathrm{C}, 90 \mathrm{~min}$; iv. $\mathrm{NaNO}_{2}$, urea, DMF, $-78{ }^{\circ} \mathrm{C}$ to $-40{ }^{\circ} \mathrm{C}$, $2.5 \mathrm{~h}$; v. Piperidine, thiophenol, $4 \AA$ powdered molecular sieves, benzene, reflux, $23 \mathrm{~h}$.

Fig. (8). Direct introduction of a three-carbon chain and attempts at an intramolecular Henry reaction.

\section{CONCLUSIONS}

The synthesis of several highly functionalized 3,4disubstituted indole building blocks was achieved from a C-4 functionalized indole derivative obtained by application of the Leimgruber-Batcho reaction. Because of their relationship with a tricyclic welwistatin fragment, these compounds contained a one-carbon functional group at C-4 and a three- 
carbon chain bearing $\omega$ alcohol, aldehyde, ketone, acetal or ester groups, while the benzylic position was functionalized by ester, hydroxy or protected hydroxy, halide or nitro groups. The compounds obtained constitute synthetically relevant materials that will hopefully find use in the preparation of bioactive indole derivatives.

\section{EXPERIMENTAL SECTION}

\section{General Information}

All reagents were of commercial quality (Aldrich, Fluka, SDS, Probus) and were used as received. Solvents (from SDS) were dried and purified using standard techniques. Reactions were monitored by thin layer chromatography, on aluminium plates coated with silica gel with fluorescent indicator (SDS CCM221254). Separations by flash chromatography were performed on silica gel (SDS 60 ACC, 230-40 mesh). Melting points were measured with a Reichert 723 hot stage microscope, and are uncorrected. Infrared spectra were recorded on a Perkin Elmer Paragon 1000 FT-IR spectrophotometer, with all compounds examined as films on a $\mathrm{NaCl}$ disk. NMR spectra were obtained on a Bruker Avance instrument (250 MHz for ${ }^{1} \mathrm{H}, 62.9 \mathrm{MHz}$ for ${ }^{13} \mathrm{C}$ ), which is maintained by CAI de Resonancia Magnética Nuclear, Universidad Complutense, with $\mathrm{CDCl}_{3}$ as solvent. Combustion elemental microanalyses were determined by CAI de Microanálisis Elemental, Universidad Complutense, using a Leco 932 CHNS microanalyzer.

\section{Ethyl 2-(2-dimethylaminovinyl)-3-nitro-benzoate (1)}

To a solution of methyl 2-methyl-3-nitrobenzoate $(4.1 \mathrm{~g}$, $20 \mathrm{mmol})$ in dimethylformamide $(16 \mathrm{~mL})$ was added $N, N$ dimethylformamide diethylacetal (10 g, $83.9 \mathrm{mmol})$. The reaction mixture was refluxed under an argon atmosphere for $48 \mathrm{~h}$, poured on water and extracted with ethyl ether $(3 \times 10$ $\mathrm{mL})$. The combined organic layers were dried over anhydrous $\mathrm{Na}_{2} \mathrm{SO}_{4}$ and evaporated, leaving a residue that was identified as compound 1 , as a reddish viscous oil $(5.025 \mathrm{~g}$, $95 \%) .{ }^{1} \mathrm{H}-\mathrm{NMR}\left(\mathrm{CDCl}_{3}, 250 \mathrm{MHz}\right) \delta: 7.82$ (m, 2H, H-6, H4); 7.15 (t, $1 \mathrm{H}, J=7.9 \mathrm{~Hz}, \mathrm{H}-5) ; 6.41(\mathrm{~d}, 1 \mathrm{H}, J=13.6 \mathrm{~Hz}$, H-2'); 5.75 (d, 1H, $\left.J=13.6 \mathrm{~Hz}, \mathrm{H}-1^{\prime}\right)$; 4.45 (q, 2H, $J=7.2$ $\left.\mathrm{Hz}, \mathrm{CH}_{2}\right) ; 2.90\left(\mathrm{~s}, 6 \mathrm{H}, \mathrm{N}\left(\mathrm{CH}_{3}\right)_{2}\right) ; 1.45(\mathrm{t}, 3 \mathrm{H}, J=7.1 \mathrm{~Hz}$, $\left.\mathrm{CH}_{3}\right) \quad$ ppm. ${ }^{13} \mathrm{C}-\mathrm{RMN} \quad\left(\mathrm{CDCl}_{3}, \quad 250 \quad \mathrm{MHz}\right) \delta: 168.8$ $\left(\mathrm{CO}_{2} \mathrm{CH}_{2} \mathrm{CH}_{3}\right) ; 149.1$ (C-3'); 146.0 (C-2'); 134.2 (C-1'); 133.1 (C-4); 132.0 (C-2'); 126.6 (C-6); 122.9 (C-5); 88.9 (C$\left.1^{\prime}\right) ; 61.4\left(\mathrm{CO}_{2} \mathrm{CH}_{2} \mathrm{CH}_{3}\right) ; 40.5\left(\mathrm{NMe}_{2}\right) ; 14.2\left(\mathrm{CO}_{2} \mathrm{CH}_{2} \mathrm{CH}_{3}\right)$ ppm. Anal. Calcd. for $\mathrm{C}_{13} \mathrm{H}_{16} \mathrm{~N}_{2} \mathrm{O}_{4}, \mathrm{M}=264$ : C, 59.08; $\mathrm{H}$, $6.10 ; \mathrm{N}, 10.60$. Found: C, 58.95; H, 5.97; N, 10.52 .

\section{Ethyl indole-4-carboxylate (2a)}

To a solution of compound $\mathbf{1}(1.597 \mathrm{~g}, 6 \mathrm{mmol})$ in methanol $(12 \mathrm{~mL})$ and tetrahydrofuran $(12 \mathrm{~mL})$ was added $80 \%$ hydrazine hydrate $(0.450 \mathrm{~g}, 9 \mathrm{mmol})$. The mixture was protected with a calcium chloride tube and stirred at room temperature while a $50 \%$ suspension of Raney $\mathrm{Ni}$ in water $(0.12 \mathrm{~mL})$ was added portionwise. The resulting suspension was stirred at room temperature and filtered through celite, which was washed with dichloromethane. The combined filtrates were dried over anhydrous $\mathrm{Na}_{2} \mathrm{SO}_{4}$ and evaporated. The residue was purified by chromatography on silica gel, eluting with a 2:1 petroleum ether-ethyl acetate mixture, to yield $907 \mathrm{mg}(80 \%)$ of indole 2. IR $(\mathrm{NaCl}) \mathrm{v}$ : $3314.8(\mathrm{NH})$; 1686.1 (CO); $1275.3 \mathrm{~cm}^{-1} .{ }^{1} \mathrm{H}-\mathrm{NMR}\left(\mathrm{CDCl}_{3}, 250 \mathrm{MHz}\right) \delta$ :
8.47 (s, 1H, NH); 7.91 (d, 1H, $J=7.5 \mathrm{~Hz}, \mathrm{H}-5) ; 7.58$ (d, 1H, $J=8.1 \mathrm{~Hz}, \mathrm{H}-7) ; 7.33(\mathrm{t}, 1 \mathrm{H}, J=3.0 \mathrm{~Hz}, \mathrm{H}-6) ; 7.24(\mathrm{~d}, 1 \mathrm{H}$, $J=5.1 \mathrm{~Hz}, \mathrm{H}-2) ; 7.18(\mathrm{~d}, 1 \mathrm{H}, J=2.3 \mathrm{~Hz}, \mathrm{H}-3) ; 4.44$ (q, 2H, $\left.J=7.1 \mathrm{~Hz}, \mathrm{CH}_{2}\right) ; 1.45\left(\mathrm{t}, 3 \mathrm{H}, J=7.1 \mathrm{~Hz}, \mathrm{CH}_{3}\right) \mathrm{ppm} .{ }^{13} \mathrm{C}-$ NMR $\left(\mathrm{CDCl}_{3}, 62.9 \mathrm{MHz}\right) \delta: 167,8\left(\mathrm{CO}_{2} \mathrm{CH}_{2} \mathrm{CH}_{3}\right) ; 136.7(\mathrm{C}-$ 7a); 127.5 (C-3a); 126.3 (C-2); 123.5 (C-5); 122.0 (C-4); 121.3 (C-6); 115.9 (C-7); 104.1 (C-3); $60.7\left(\mathrm{CO}_{2} \mathrm{CH}_{2} \mathrm{CH}_{3}\right)$; $14,58\left(\mathrm{CO}_{2} \mathrm{CH}_{2} \mathrm{CH}_{3}\right)$ ppm. Analysis: Calcd. for $\mathrm{C}_{11} \mathrm{H}_{11} \mathrm{NO}_{2}$, $\mathrm{M}=189$ : C, 69.83; H, 5.86; N, 7.40. Found: C, 69.56; H, $5.44 ; \mathrm{N}, 7.40$.

\section{Ethyl 1-methylindole-4-carboxylate (2b)}

A commercial suspension of sodium hydride in paraffins $(60 \%, 138 \mathrm{mg}, 5.76 \mathrm{mmol})$ was washed with dry petroleum ether $(3 \times 10 \mathrm{~mL})$, under an argon atmosphere. A solution of compound 2 (907 mg, $4.8 \mathrm{mmol}$ ) in dry dimethylformamide ( $3 \mathrm{~mL}$ ) was added, and the resulting suspension was stirred at room temperature for $5 \mathrm{~min}$. Methyl iodide (2 g, 14.4 mmol) was added and stirring at room temperature was maintained for $1 \mathrm{~h}$. The reaction mixture was poured on crushed ice $(10 \mathrm{~g})$ and extracted with ethyl acetate $(3 \times 10$ $\mathrm{mL})$. The combined organic layers were dried over anhydrous $\mathrm{Na}_{2} \mathrm{SO}_{4}$ and evaporated, and the residue was chromatographed on silica gel, eluting with a $2: 1$ petroleum etherethyl acetate mixture, to yield $850 \mathrm{~g}(87 \%)$ of indole 3 . IR $(\mathrm{NaCl}) \mathrm{v}: 1702,5(\mathrm{CO}) \mathrm{cm}^{-1} .{ }^{1} \mathrm{H}-\mathrm{NMR}\left(\mathrm{CDCl}_{3}, 250 \mathrm{MHz}\right) \delta$ : $7.91(\mathrm{~d}, 1 \mathrm{H}, J=7.5 \mathrm{~Hz}$; H-5); $7.51(\mathrm{~d}, 1 \mathrm{H}, J=8.2 \mathrm{~Hz}, \mathrm{H}-7)$; 7.25 (t, 1H, $J=8.4 \mathrm{~Hz}, \mathrm{H}-6) ; 7.17$ (d, 1H, $J=3.1 \mathrm{~Hz}, \mathrm{H}-2)$; $7.09(\mathrm{~d}, 1 \mathrm{H}, J=3.1 \mathrm{~Hz}, \mathrm{H}-3) ; 4.44(\mathrm{q}, 2 \mathrm{H}, J=14.2 \mathrm{~Hz}$, $\left.\mathrm{CH}_{2}\right) ; 3.82\left(\mathrm{~s}, 3 \mathrm{H}, \mathrm{CH}_{3}\right) ; 1.45\left(\mathrm{t}, 3 \mathrm{H}, J=7.1 \mathrm{~Hz}, \mathrm{CH}_{3}\right) \mathrm{ppm}$. ${ }^{13} \mathrm{C}-\mathrm{NMR}\left(\mathrm{CDCl}_{3}, 62.9 \mathrm{MHz}\right) \delta$ : $167.8(\mathrm{CO}) ; 137.6(\mathrm{C}-7 \mathrm{a})$; 130.9 (C-3a); 128.1 (C-2); 123.0 (C-5); 122.0 (C-4); 120.7 (C-6); 114.0 (C-7); 102.4 (C-3); $60.6\left(\mathrm{CO}_{2} \underline{\mathrm{CH}_{2}} \mathrm{CH}_{3}\right) ; 33,1$ $\left(\mathrm{N}-\mathrm{CH}_{3}\right) ; 14.6\left(\mathrm{CO}_{2} \mathrm{CH}_{2} \mathrm{CH}_{3}\right)$ ppm. Analysis: Calcd. for $\mathrm{C}_{12} \mathrm{H}_{13} \mathrm{NO}_{2}, \mathrm{M}=203$ : C, 70.92; H-6.45; N, 6.89. Found: C$70.80 ; \mathrm{H}, 6.45 ; \mathrm{N}, 6.80$.

\section{(Indol-4-yl)methanol (3a)}

To a stirred suspension of lithium aluminium hydride $(115 \mathrm{mg}, 3 \mathrm{mmol})$ in ethyl ether $(10 \mathrm{~mL})$ and tetrahydrofuran $(10 \mathrm{~mL})$, kept under an argon atmosphere and cooled to 0 ${ }^{\circ} \mathrm{C}$, was added dropwise a solution of $2 \mathbf{a}$ (370 mg, 2.11 $\mathrm{mmol})$ in the same solvent mixture $(10 \mathrm{~mL})$. The suspension was stirred at room temperature for $48 \mathrm{~h}$. Excess lithium aluminium hydride was destroyed by addition of ethyl acetate followed by a few drops of water, maintaining initially the reaction mixture in a bath at $0{ }^{\circ} \mathrm{C}$ and then at room temperature for $30 \mathrm{~min}$. Solid sodium bicarbonate was then added until a white solid was formed, which was filtered and washed with ethyl acetate. The filtrate was dried over anhydrous $\mathrm{Na}_{2} \mathrm{SO}_{4}$ and evaporated, and the pale brown residue was identified as alcohol 3a (234 mg, 93\%). Anal. Calcd. for $\mathrm{C}_{9} \mathrm{H}_{9} \mathrm{NO}, \mathrm{M}=147: \mathrm{C}, 73.45 ; \mathrm{H}, 6.16 ; \mathrm{N}, 9.52$. Found: $\mathrm{C}$, 73.10; H, 6.40; N, 9.23.

\section{(1-Methylindol-4-yl)methanol (3b)}

The same method described for the preparation of $\mathbf{3 a}$, starting from $439 \mathrm{mg}$ (2.32 mmol) of compound $\mathbf{2 b}$, afforded $330 \mathrm{mg}(88 \%)$ of alcohol 3b. IR $(\mathrm{NaCl}) \mathrm{v}: 3363.7(\mathrm{OH}) \mathrm{cm}^{-1}$. ${ }^{1} \mathrm{H}-\mathrm{NMR}\left(\mathrm{CDCl}_{3}, 250 \mathrm{MHz}\right) \delta:$ 7,30-7,10 (m, 3H, H-5, H-6, $\mathrm{H}-7) ; 7.08$ (d, $1 \mathrm{H}, J=3.2 \mathrm{~Hz}, \mathrm{H}-2) ; 6.59$ (d, $1 \mathrm{H}, J=3.2 \mathrm{~Hz}$, $\mathrm{H}-3) ; 4.97$ (s, 2H, C $\left.4-\mathrm{CH}_{2}\right) ; 3.80\left(\mathrm{~s}, 3 \mathrm{H}, \mathrm{N}-\mathrm{CH}_{3}\right) ; 1.67$ (s, 1H, $\mathrm{OH})$ ppm. ${ }^{13} \mathrm{C}-\mathrm{NMR}\left(\mathrm{CDCl}_{3}, 62.9 \mathrm{MHz}\right) \delta: 136.9$ (C-7a); 
132.9 (C-4); 129.1 (C-2); 126.8 (C-3a); 121.7 (C-5); 118.0 (C-6); 109.2 (C-7); 99.1 (C-3); $64.1\left(\mathrm{C}_{4}-\mathrm{CH}_{2}\right) ; 33.1\left(\mathrm{~N}-\mathrm{CH}_{3}\right)$ ppm. Anal. Calcd. for $\mathrm{C}_{10} \mathrm{H}_{11} \mathrm{NO}, \mathrm{M}=161$ : $\mathrm{C}, 74.51 ; \mathrm{H}$, $6.88 ;$ N, 8.69. Found: C, 74.38; H, 6.74; N, 8.32.

\section{4-(tert-Butyldiphenylsilyloxy)indole (4a)}

To a solution of indole 3a $(68 \mathrm{mg}, 0.463 \mathrm{mmol})$ in dry dichlorometane $(0.6 \mathrm{~mL})$, kept under an argon atmosphere and cooled at $0{ }^{\circ} \mathrm{C}$, was added triethylamine $(65 \mu \mathrm{L}, 0.55$ mmol). After stirring for $5 \mathrm{~min}$ at $0{ }^{\circ} \mathrm{C}$, tertbutyldiphenylsilyl chloride $(140 \mu \mathrm{L}, 0.55 \mathrm{mmol})$ and $4-$ dimethylaminopyridine $(25 \mathrm{mg}, 0.2 \mathrm{mmol})$ were added. The reaction mixture was left to warm to room temperature and stirred for $12 \mathrm{~h}$, poured onto water $(10 \mathrm{~mL})$ and extracted with chloroform $(3 \times 10 \mathrm{~mL})$. The combined organic layers were dried over anhydrous $\mathrm{Na}_{2} \mathrm{SO}_{4}$ and evaporated, leaving a residue that was chromatographed on silica gel, eluting with a 10:1 petroleum ether-ethyl acetate mixture, to yield $126 \mathrm{mg}$ (71\%) of compound $\mathbf{4 a}$, as a colourless viscous oil. IR (NaCl) v: $3316.8(\mathrm{NH}) \mathrm{cm}^{-1} .{ }^{1} \mathrm{H}-\mathrm{NMR}\left(\mathrm{CDCl}_{3}, 250 \mathrm{MHz}\right) \delta$ : 8.12 (br s, $1 \mathrm{H}, \mathrm{NH}) ; 7.77$ (m, 4H, H-2",6”); 7.44-7.25 (m, 8H, H-7,5,3",4",5"); 7.20 (t, 1H, J = 7.5 Hz, H-6); 7.15 (t, $1 \mathrm{H}, J=2.8 \mathrm{~Hz}, \mathrm{H}-2) ; 6.52$ (m, 1H, H-3); 5.11 (s, 2H, H- $\alpha$ ); 1.12 (s, 9H, C $\left.\left(\mathrm{CH}_{3}\right)_{3}\right)$ ppm. ${ }^{13} \mathrm{C}-\mathrm{NMR}\left(\mathrm{CDCl}_{3}, 62.9 \mathrm{MHz}\right) \delta$ : 135.8 (C-7a); 135.8 (C-2",6”); 133.8 (C-1"); 133.1 (C-4); 129.8 (C-4”); 129.7 (C-2); 127.9 (C-3”,5”); 123.9 (C-3a); 122.1 (C-5); 117.0 (C-6); 110.1 (C-7); 100.9 (C-3); 64.5 (C$\alpha) ; 27.03\left(\mathrm{C}\left(\underline{\mathrm{CH}}_{3}\right)_{3}\right) ; 19.7\left(\underline{\mathrm{C}}\left(\mathrm{CH}_{3}\right)_{3}\right)$ ppm. Anal. Calcd. for $\mathrm{C}_{25} \mathrm{H}_{27} \mathrm{NOSi}, \mathrm{M}=385$ : C, 77.88; H, 7.06; N, 3.63. Found: C, 77.57; H, 7.03; N, 3.52 .

\section{4-(tert-Butyldiphenylsilyloxy)-1-methylindole (4b)}

The same method described for the preparation of $\mathbf{4 a}$, starting from $4.845 \mathrm{~g}, 30 \mathrm{mmol}$ ) of compound $\mathbf{3 b}$, afforded $9.470 \mathrm{~g}(79 \%)$ of $\mathbf{4 b}$, as a white solid. $\mathrm{Mp} 60{ }^{\circ} \mathrm{C}$. IR $(\mathrm{NaCl})$ v: $1111.8(\mathrm{C}-\mathrm{O}) \mathrm{cm}^{-1} .{ }^{1} \mathrm{H}-\mathrm{NMR}\left(\mathrm{CDCl}_{3}, 250 \mathrm{MHz}\right) \delta: 7.75$ (m, 4H, H-2",6"); 7.43-7.34 (m, 6H, H-,3",4",5"); 7.25-7.23 (m, 3H, H-5,6,7); 7.01 (d, 1H, $J=2.5 \mathrm{~Hz}, \mathrm{H}-3) ; 6.42(\mathrm{~d}, 1 \mathrm{H}$, $J=5.0 \mathrm{~Hz}, \mathrm{H}-2) ; 5.07$ (s, 2H, H- $\alpha) ; 3.78\left(\mathrm{~s}, 3 \mathrm{H}, \mathrm{N}-\mathrm{CH}_{3}\right)$; $1.10\left(\mathrm{~s}, 9 \mathrm{H}, \mathrm{C}\left(\mathrm{CH}_{3}\right)_{3}\right)$ ppm. ${ }^{13} \mathrm{C}-\mathrm{NMR}\left(\mathrm{CDCl}_{3}, 62.9 \mathrm{MHz}\right) \delta$ : 136.7 (C-7a); 135.8 (C-2",6”); 133.9 (C-1"); 133.1 (C-4); 129.7 (C-4"); 128.6 (C-2); 127.8 (C-3",5"); 125.9 (C-3a); 121.6 (C-5); 116.6 (C-6); 108.3 (C-7); 99.2 (C-3); 64.4 (C$\alpha)$; $33.1\left(\mathrm{~N}-\mathrm{CH}_{3}\right) ; 27.0\left(\mathrm{C}\left(\underline{\mathrm{CH}}_{3}\right)_{3}\right) ; 19.6\left(\underline{\mathrm{C}}\left(\mathrm{CH}_{3}\right)_{3}\right)$ ppm. Anal. Calcd. for $\mathrm{C}_{26} \mathrm{H}_{29} \mathrm{NOSi}$ : C, 78.15; H, 7.31; N, 3.51. Found: 77.89; H, 7.13; N, 3.42.

\section{(3-(Dimethylaminomethyl)indol-4-yl)methanol (5a)}

To a cooled $\left(0{ }^{\circ} \mathrm{C}\right)$ solution of compound 3a $(235 \mathrm{mg}, 1.6$ $\mathrm{mmol})$ in glacial acetic acid $(0.8 \mathrm{~mL})$, maintained under an argon atmosphere, was added a $37 \%$ aqueous solution of formaldehyde $(0.13 \mathrm{~mL}, 1.6 \mathrm{mmol})$ and a $25 \%$ aqueous solution of dimethylamine $(0.18 \mathrm{~mL}, 1.6 \mathrm{mmol})$. The solution was left to warm to room temperature and stirred for $1.5 \mathrm{~h}$, poured onto $30 \%$ aqueous sodium hydroxide $(10 \mathrm{~mL})$ and extracted with chloroform $(3 \times 10 \mathrm{~mL})$. The combined organic layers were dried over anhydrous $\mathrm{Na}_{2} \mathrm{SO}_{4}$ and evaporated, yielding $298 \mathrm{mg}(92 \%)$ of compound $\mathbf{5 a}$ as a pale green, viscous oil. IR $(\mathrm{NaCl})$ v: 3210.5 (broad signal, $\mathrm{NH}$ and $\mathrm{OH}) .{ }^{1} \mathrm{H}-\mathrm{NMR}\left(\mathrm{CDCl}_{3}, 250 \mathrm{MHz}\right) \delta: 8.28(\mathrm{~s}, 1 \mathrm{H}, \mathrm{NH})$; $7.26(\mathrm{~d}, 1 \mathrm{H}, J=7.5 \mathrm{~Hz}$; H-7); 7.13 (t, $1 \mathrm{H}, J=7.5 \mathrm{~Hz}, \mathrm{H}-6)$; 7.05 (d, 1H, $J=1.0 \mathrm{~Hz}, \mathrm{H}-5)$; 6.97 (d, 1H, $J=7.5 \mathrm{~Hz}, \mathrm{H}-2)$; 4.84 (s, 2H, H- $\alpha) ; 3.76$ (s, 2H, H- $\alpha$ '); 2.24 (s, 6H, N( $\left.\left.\mathrm{CH}_{3}\right)_{2}\right)$ ppm. ${ }^{13} \mathrm{C}-\mathrm{NMR}\left(\mathrm{CDCl}_{3}, 62.9 \mathrm{MHz}\right) \delta$ : 138.0 (C-7a); 135.0 (C-4); 124.3 (C-3a); 125.3 (C-2); 122.4 (C-5); 121.1 (C-6); 112.1 (C-3); 111.6 (C-7); 68.6 (C- $\alpha) ; 57.4$ (C- $\left.\alpha^{\prime}\right) ; 44.5$ $\left(\mathrm{N}\left(\mathrm{CH}_{3}\right)_{2}\right)$ ppm. Anal. Calcd. for $\mathrm{C}_{12} \mathrm{H}_{16} \mathrm{~N}_{2} \mathrm{O}, \mathrm{M}=204$ : C, 70.56; H, 7.90; N, 13.71. Found: C, 70.23; H, 7.64; N, 13.39.

\section{(3-(Dimethylaminomethyl)-1-methylindol-4-yl)methanol (5b)}

The same method described for the preparation of $\mathbf{5 a}$, starting from $400 \mathrm{mg}$ (3.1 mmol) of compound $\mathbf{3 b}$, afforded $438 \mathrm{mg}(86 \%)$ of $\mathbf{5 b} .{ }^{1} \mathrm{H}-\mathrm{NMR}\left(\mathrm{CDCl}_{3}, 250 \mathrm{MHz}\right) \delta: 7.22(\mathrm{~d}$, $1 \mathrm{H}, J=7.1 \mathrm{~Hz}, \mathrm{H}-7) ; 7.15$ (t, $1 \mathrm{H}, J=6.9 \mathrm{~Hz}, \mathrm{H}-6) ; 6.99(\mathrm{~d}$, $1 \mathrm{H}, J=6.9 \mathrm{~Hz}, \mathrm{H}-5) ; 6.91(\mathrm{~s}, 1 \mathrm{H}, \mathrm{H}-2) ; 4.84(\mathrm{~d}, 2 \mathrm{H}$, $\left.\mathrm{CH}_{2} \mathrm{OH}\right) ; 3.73\left(\mathrm{~s}, 5 \mathrm{H}, \mathrm{N}-\mathrm{CH}_{3}\right.$ and $\left.\mathrm{CH}_{2}-\mathrm{N}\left(\mathrm{CH}_{3}\right)_{2}\right) ; 2.24(\mathrm{~s}$, $\left.6 \mathrm{H}, \mathrm{N}\left(\mathrm{CH}_{3}\right)_{2}\right)$ ppm. ${ }^{13} \mathrm{CNMR}\left(\mathrm{CDCl}_{3}, 62.9 \mathrm{MHz}\right) \delta: 138.6$ (C-7a); 135.6 (C-4); 129.6 (C-2); 124.8 (C-3a); 122.2 (C-5); 120.9 (C-6); 111.1 (C-3); 109.6 (C-7); $64.4\left(\mathrm{CH}_{2} \mathrm{OH}\right) ; 44.7$ $\left(\mathrm{N}\left(\mathrm{CH}_{3}\right)_{2} ; 32.9\left(\mathrm{~N}-\mathrm{CH}_{3}\right)\right.$ ppm. Anal. Calcd. for $\mathrm{C}_{13} \mathrm{H}_{18} \mathrm{~N}_{2} \mathrm{O}$, $\mathrm{M}=218: \mathrm{C}, 71.53 ; \mathrm{H}, 8.31 ; \mathrm{N}, 12.83$. Found: $\mathrm{C}, 71.26 ; \mathrm{H}$, $7.97 ; \mathrm{N}, 12.46$.

\section{(4-Hydroxymethyl-3-indolylmethyl) trimethylamonium io- dide (6a)}

To a cooled $\left(0{ }^{\circ} \mathrm{C}\right)$, stirred solution of compound 5a (200 $\mathrm{mg}, 0.98 \mathrm{mmol})$ in dry tetrahydrofuran $(4 \mathrm{~mL})$, maintained under an argon atmosphere, was slowly added neat methyl iodide $(2.75 \mathrm{~mL})$, maintaining the temperature of the bath at $0{ }^{\circ} \mathrm{C}$. The resulting solution was stirred at room temperature for $30 \mathrm{~min}$ and then kept at $4{ }^{\circ} \mathrm{C}$ for $14 \mathrm{~h}$ and filtered, affording $235 \mathrm{mg}(82 \%)$ of salt $\mathbf{6 a}$, as a pale brown solid. ${ }^{1} \mathrm{H}-$ NMR $\left(\mathrm{CDCl}_{3}, 250 \mathrm{MHz}\right) \delta: 7.88$ (s, 1H, H-2); 7.77 (d, 1H, J $=7.5 \mathrm{~Hz}, \mathrm{H}-7) ; 7.47$ (t, $1 \mathrm{H}, J=7.5 \mathrm{~Hz}, \mathrm{H}-6) ; 7.33$ (d, $1 \mathrm{H}, J$ $=7.0 \mathrm{~Hz}, \mathrm{H}-5) ; 5.34\left(\mathrm{~s}, 2 \mathrm{H}, \mathrm{CH}_{2}-\mathrm{N}\right) ; 4.97\left(\mathrm{~s}, 2 \mathrm{H}, \mathrm{CH}_{2} \mathrm{OH}\right)$; 3.24 (s, 9H, N( $\left.\left.\mathrm{CH}_{3}\right)_{3}\right)$ ppm. Anal. Calcd. for $\mathrm{C}_{13} \mathrm{H}_{19} \mathrm{IN}_{2} \mathrm{O}, \mathrm{M}$ = 346: C, 45.10; H, 5.53; N, 8.09. Found: C, 44.81; H, 5.32; $\mathrm{N}, 5.26$.

\section{(4-Hydroxymethyl-1-methyl-3-indolylmethyl) trimethylam- monium iodide $(6 \mathrm{~b})$}

To a cooled $\left(0{ }^{\circ} \mathrm{C}\right)$, stirred solution of compound $\mathbf{5 a}$ (565 $\mathrm{mg}, 2.6 \mathrm{mmol})$ in dry tetrahydrofuran $(10 \mathrm{~mL})$, maintained under an argon atmosphere, was slowly added neat methyl iodide $(7.3 \mathrm{~mL})$, maintaining the temperature of the bath at 0 ${ }^{\circ} \mathrm{C}$. The resulting solution was stirred at room temperature for $30 \mathrm{~min}$ and then kept at $4{ }^{\circ} \mathrm{C}$ for $14 \mathrm{~h}$ and filtered. The precipitate was washed with methanol, leaving an insoluble residue. The methanol washings were evaporated, affording $410 \mathrm{mg}(43 \%)$ of salt $\mathbf{6 b}$, as a pale orange solid. Mp $60{ }^{\circ} \mathrm{C}$. ${ }^{1} \mathrm{H}-\mathrm{NMR}\left(\mathrm{CDCl}_{3}, 250 \mathrm{MHz}\right) \delta: 7.57$ (s, 1H, H-2); 7.24 (overlapped with $\left.\mathrm{CHCl}_{3}, \mathrm{H}-7\right)$; 7.19 (t, $\left.1 \mathrm{H}, J=7.1 \mathrm{~Hz}, \mathrm{H}-6\right)$; $7.10(\mathrm{~d}, 1 \mathrm{H}, J=6.8 \mathrm{~Hz}, \mathrm{H}-5) ; 5.18\left(\mathrm{~s}, 2 \mathrm{H}, \mathrm{CH}_{2}-\mathrm{N}\right) ; 4.95$ (s, $\left.2 \mathrm{H}, \mathrm{CH}_{2}-\mathrm{OH}\right) ; 3.73\left(\mathrm{~s}, 3 \mathrm{H}, \mathrm{CH}_{3}\right) ; 3,22\left(\mathrm{~s}, 9 \mathrm{H}, \mathrm{N}\left(\mathrm{CH}_{3}\right)_{3}\right)$ ppm. Anal. Calcd. for $\mathrm{C}_{14} \mathrm{H}_{21} \mathrm{IN}_{2} \mathrm{O}, \mathrm{M}=360$ : C, 46.68; $\mathrm{H}$, 5.88 ; N, 7.78. Found: C, 46.38; H, 5.68; N, 7.32.

\section{Ethyl 2-((4-hydroxymethyl-1-methyl-3-indolyl)methyl)mal- onate (7)}

To an ethanolic solution of sodium ethoxide, freshly prepared from sodium (50 mg, $2.18 \mathrm{mmol}$ ) in absolute ethanol (3.3 mL), was added freshly distilled diethyl malonate (347 $\mathrm{mg}, 2.18 \mathrm{mmol}$ ) and the solution was stirred at room temperature for $15 \mathrm{~min}$, under an argon atmosphere. A solution of compound $\mathbf{6 b}(239 \mathrm{mg}, 0.664 \mathrm{mmol})$ in absolute ethanol ( $2 \mathrm{~mL}$ ) was prepared by brief reflux, cooled and added to the 
sodium diethyl malonate solution. The reacting mixture was refluxed under an argon atmosphere for $12 \mathrm{~h}$, cooled and evaporated to dryness. The residue was dissolved in water (5 $\mathrm{mL})$ and the solution was extracted with chloroform ( $3 \times 10$ $\mathrm{mL}$ ). The remaining aqueous phase was acidified to $\mathrm{pH} 4$ with $36 \% \mathrm{HCl}$ and extracted with chloroform $(4 \times 10 \mathrm{~mL})$. The combined chloroform phases from the second extraction were dried over anhydrous $\mathrm{Na}_{2} \mathrm{SO}_{4}$ and evaporated, leaving a residue that was purified by chromatography on silica gel, eluting with a petroleum ether-ethyl acetate gradient, to yield $50 \mathrm{mg}(23 \%)$ of compound 7. ${ }^{1} \mathrm{H}-\mathrm{NMR}\left(\mathrm{CDCl}_{3}, 250\right.$ MHz) $\delta$ : 7.24 (m, H-7'); 7,14 (t, 1H, $\left.J=6.9 \mathrm{~Hz}, \mathrm{H}-6^{\prime}\right) ; 7.02$ (d, 1H, $J=6.7 \mathrm{~Hz}, \mathrm{H}-5$ '), 6.93 (s, 1H, H-2'); 4.99 (q, 2H, $J$ $\left.=12.2 \mathrm{~Hz}, \mathrm{CH}_{2} \mathrm{OH}\right) ; 4.20\left(\mathrm{~m}, 4 \mathrm{H}, 2 \mathrm{CH}_{2}\right) ; 3,91(\mathrm{t}, 1 \mathrm{H}, J=$ $7.2 \mathrm{~Hz}, \mathrm{H}-2) ; 3.69\left(\mathrm{~s}, 3 \mathrm{H}, \mathrm{N}_{1},-\mathrm{CH}_{3}\right) ; 3.53(\mathrm{~d}, 2 \mathrm{H}, J=6.1 \mathrm{~Hz}$, $\mathrm{H}-\alpha) ; 1.23\left(\mathrm{~m}, 6 \mathrm{H}, 2 \mathrm{CH}_{3}\right)$ ppm. ${ }^{13} \mathrm{C}-\mathrm{NMR}\left(\mathrm{CDCl}_{3}, 62.9\right.$ $\mathrm{MHz}) \delta$ : $167.4\left(\mathrm{CO}_{2} \mathrm{CH}_{2} \mathrm{CH}_{3}\right) ; 137.6\left(\mathrm{C}-7 \mathrm{a}^{\prime}\right) ; 132.1$ (C-4'); 128.6 (C-2'); 124.9 (C-3a'); 121.3 (C-5'); 120.5 (C-6'); 110.1 $\left(\mathrm{C}-3^{\prime}\right) ; 109.9$ (C-7'); $64.2\left(\mathrm{CH}_{2} \mathrm{OH}\right) ; 61.9$ and $61.6(2$ $\left(\mathrm{CO}_{2} \mathrm{CH}_{2} \mathrm{CH}_{3}\right) ; 53.5(\mathrm{C}-2) ; 40.4(\mathrm{C}-\alpha) ; 32.7\left(\mathrm{~N}^{-} \mathrm{CH}_{3}\right) ; 13.9$ and 13.9 $\left(\mathrm{CO}_{2} \mathrm{CH}_{2} \mathrm{CH}_{3}\right) \mathrm{ppm}$. Anal. Calcd. for $\mathrm{C}_{18} \mathrm{H}_{23} \mathrm{NO}_{5}, \mathrm{M}$ = 333: C, 64.85; H, 6.95; N, 4.20. Found: C, 64.58; H, 6.78; $\mathrm{N}, 4.07$.

\section{3-Dimethylamino-4-(tert-butyldiphenylsilyloxymethyl) indole (8)}

To a solution of alcohol $\mathbf{5 b}(67 \mathrm{mg}, 0.328 \mathrm{mmol})$ in dry dichloromethane $(0.5 \mathrm{~mL})$, cooled to $0{ }^{\circ} \mathrm{C}$ and in an argon atmosphere, was added triethylamine $(50 \mu \mathrm{L}, 0.39 \mathrm{mmol})$. The mixture was stirred for $5 \mathrm{~min}$ at $0{ }^{\circ} \mathrm{C}$ and tertbutyldiphenylsilyl chloride $(100 \mu \mathrm{L}, 0.39 \mathrm{mmol})$ and 4 dimethylaminopyridine $(25 \mathrm{mg}, 0.2 \mathrm{mmol})$ were added. The reaction was left to reach room temperature and stirring was maintained for $12 \mathrm{~h}$. The reaction mixture was poured on water $(10 \mathrm{~mL})$ and extracted with chloroform $(3 \times 10 \mathrm{~mL})$. The combined organic layers were dried over anhydrous $\mathrm{Na}_{2} \mathrm{SO}_{4}$ and evaporated, leaving a residue that was purified by chromatography on silica gel, eluting with a $8: 1$ mixture of petroleum ether and ethyl acetate, affording $96 \mathrm{mg}(66 \%)$ of compound 8, as a white solid. IR ( NaCl) v: $3064(\mathrm{NH})$ $\mathrm{cm}^{-1} .{ }^{1} \mathrm{H}-\mathrm{NMR}\left(\mathrm{CDCl}_{3}, 250 \mathrm{MHz}\right) \delta: 8.02(\mathrm{~s}, 1 \mathrm{H}, \mathrm{NH}) ; 7.72$ (dd, $4 \mathrm{H}, J=7.5$ and $2.5 \mathrm{~Hz}, \mathrm{H}-2$ ", $6 ")$ ) 7.43-7.26 (m, 8H, H5,7,3",4",5"); 7.16 (t, 1H, J = 7.5 Hz, H-6); 7.01 (s, 1H, H2); 5.36 (s, 2H, H- $\alpha$ ); 3.38 (s, 2H, H- $\left.\alpha^{\prime}\right) ; 1.99$ (s, 6H, $\left.\mathrm{N}\left(\mathrm{CH}_{3}\right)_{2}\right) ; 1.09$ (s, 9H, $\left.\mathrm{C}\left(\mathrm{CH}_{3}\right)_{3}\right)$ ppm. Anal. Calcd. for $\mathrm{C}_{28} \mathrm{H}_{34} \mathrm{~N}_{2} \mathrm{OSi}, \mathrm{M}=442$ : C, 75.97; H, 7.74; N, 6.33. Found: C, 75.95; H, 7.58; N, 6.30 .

\section{Diethyl 2-((4'-tert-butyldiphenylsilyloxymethyl-1'-methyl- 3 '-indolyl)methyl) malonate (9)}

To a solution of compound 8 (50 mg, 0,113 mmol) in dry tetrahydrofuran $(2 \mathrm{~mL})$, under an inert atmosphere, was added a solution of tributylphosphine $(28 \mu \mathrm{L}, 0.113 \mathrm{mmol})$ in dry tetrahydrofuran $(1 \mathrm{~mL})$. The mixture was stirred for 5 min and diethyl malonate $(30 \mu \mathrm{L}, 0,136 \mathrm{mmol})$ was added. The reaction mixture was refluxed for $24 \mathrm{~h}$, cooled and poured on water $(5 \mathrm{~mL})$ containing a few drops of $0.5 \mathrm{M}$ aqueous $\mathrm{HCl}$. After extraction with a 95:5 dichloromethanemethanol mixture $(3 \times 5 \mathrm{~mL})$, the combined organic layers were washed with brine $(10 \mathrm{~mL})$ dried over anhydrous $\mathrm{Na}_{2} \mathrm{SO}_{4}$ and evaporated. The residue was chomatographed on silica gel, eluting with a 1:1 petroleum ether-ethyl acetate mixture, affording $30 \mathrm{mg}$ (48\%) of compound 9 as a pale yellow oil. IR (NaCl) v: $3404.0(\mathrm{NH}) ; 1730.3(\mathrm{CO}) \mathrm{cm}^{-1} .{ }^{1} \mathrm{H}-$ NMR $\left(\mathrm{CDCl}_{3}, 250 \mathrm{MHz}\right) \delta: 8.03(\mathrm{~s}, 1 \mathrm{H}, \mathrm{NH}) ; 7.66(\mathrm{dd}, 4 \mathrm{H}$, $J=7.5$ and $2.5 \mathrm{~Hz}, \mathrm{H}-2 ", 6 ") ; 7.43-7.23$ (m, 8H, H5',7',3”, 4",5”); 7.07 (t, $1 \mathrm{H}, J=7.5 \mathrm{~Hz}, \mathrm{H}-6$ '); 6.99 (s, 1H, $\mathrm{H}-2$ ); 5.14 (s, $2 \mathrm{H}, \mathrm{H}-\alpha$ ); 4.07 (q, $2 \mathrm{H}, J=7.5 \mathrm{~Hz}, \mathrm{C}_{2} \mathrm{CH}_{3}$ ); 3.72 (t, $2 \mathrm{H}, J=7.5 \mathrm{~Hz}, \mathrm{H}-2) ; 3.51$ (d, $\left.2 \mathrm{H}, J=7.5 \mathrm{~Hz}, \mathrm{H}-\alpha^{\prime}\right)$; $1.12\left(\mathrm{t}, 3 \mathrm{H}, J=7.5 \mathrm{~Hz}, \mathrm{CH}_{2} \mathrm{CH}_{3}\right) ; 1.04\left(\mathrm{~s}, 9 \mathrm{H}, \mathrm{C}\left(\mathrm{CH}_{3}\right)_{3}\right)$ ppm. ${ }^{13} \mathrm{C}-\mathrm{NMR}\left(\mathrm{CDCl}_{3}, 62.9 \mathrm{MHz}\right) \delta: 169.3(\mathrm{CO}) ; 136.9(\mathrm{C}-$ 7a'); 135.8 (C-2",6”); 133.8 (C-1"); 133.1 (C-4'); 129.7 (C4"); 127.7 (C-3",5"); 124.5 (C-3a'); 123.2 (C-2'); 121.9 (C5'); 119.3 (C-6'); 112.8 (C-3'); 110.8 (C-7'); 64.8 (C- $\alpha$ ); $61.7\left(\mathrm{CH}_{2} \mathrm{CH}_{3}\right) ; 53.7(\mathrm{C}-2) ; 27.0\left(\mathrm{C}\left(\mathrm{CH}_{3}\right)_{3}\right) ; 26.3(\mathrm{C}-\alpha)$; $19.4\left(\underline{\mathrm{C}}\left(\mathrm{CH}_{3}\right)_{3}\right) ; 14.1\left(\mathrm{CH}_{2} \mathrm{CH}_{3}\right)$ ppm. Anal. Calcd. for $\mathrm{C}_{33} \mathrm{H}_{39} \mathrm{NO}_{5} \mathrm{Si}, \mathrm{M}=557: \mathrm{C}, 71.06 ; \mathrm{H}, 7.05 ; \mathrm{N}, 2.51$. Found: C, 70.86; H, 6.86; N, 2.35 .

\section{(4'-tert-Butyldiphenylsilyloxymethyl-1'-methylindol-3'-yl) methylene)dimethyl-ammonium chloride (10)}

A solution of phosphorous oxychloride $(0.3 \mathrm{~mL}, 3.05$ mmol) in anhydrous dimethylformamide $(0.7 \mathrm{~mL})$ was stirred at $0{ }^{\circ} \mathrm{C}$ for 30 min under an argon atmosphere. A solution of compound $\mathbf{4 b}(976 \mathrm{mg}, 2.45 \mathrm{mmol})$ in anhydrous dimethylformamide $(5 \mathrm{~mL})$ was added dropwise. The reaction mixture was stirred at room temperature for $45 \mathrm{~min}$ and poured on water $(10 \mathrm{~mL})$. After stirring for $30 \mathrm{~min}$, the mixture was cooled to $0{ }^{\circ} \mathrm{C}$, giving a precipitate that was filtered and dried under reduced pressure in the presence of phosphorous pentoxide to yield $973 \mathrm{mg}(80 \%)$ of compound 10, as a white solid. Mp 128-129 ${ }^{\circ} \mathrm{C} .{ }^{1} \mathrm{H}-\mathrm{NMR}\left(\mathrm{CDCl}_{3}, 250\right.$ $\mathrm{MHz}) \delta$ : 9.22 (s, 1H, H- $\left.\alpha^{\prime}\right) ; 7.54$ (d, 4H, $J=7.5 \mathrm{~Hz}, \mathrm{H}-$ 2",6"); 7.47-7.19 (m, 9H, H-5,6,7,3",4",5"); 6.82 (d, 1H, $J=$ $7.5 \mathrm{~Hz}, \mathrm{H}-2) ; 5.00$ (s, 2H, H- $\alpha$ ); 4.13 (s, 3H, N' $\mathrm{CH}_{3}$ ); 3.86 $\left(\mathrm{s}, 3 \mathrm{H}, \mathrm{N}-\mathrm{CH}_{3}\right) ; 3.55\left(\mathrm{~s}, 3 \mathrm{H}, \mathrm{N}^{\prime}-\mathrm{CH}_{3}\right) ; 0,96\left(\mathrm{~s}, 9 \mathrm{H}, \mathrm{C}\left(\mathrm{C}_{3}\right)_{3}\right)$ ppm ${ }^{13} \mathrm{C}-\mathrm{NMR}\left(\mathrm{CDCl}_{3}, 62.9 \mathrm{MHz}\right) \delta: 160.6\left(\mathrm{C}^{\prime} \alpha^{\prime}\right) ; 142.4$ (C-2); 138.3 (C-7a); 135.6 (C-2",6"); 132.7 (C-1"); 131.9 (C-4); 130.3 (C-4"); 128.1 (C-3",5"); 126.7 (C-3a); 125.5 (C-5); 124.6 (C-6); 111.8 (C-7); 105.9 (C-3); 65.9 (C- $\alpha$ ); 50.4 and $44.2\left(2 \mathrm{~N}^{\prime}-\mathrm{CH}_{3}\right) ; 35.0\left(\mathrm{~N}^{-\mathrm{CH}_{3}}\right) ; 26.9\left(\mathrm{C}\left(\mathrm{CH}_{3}\right)_{3}\right)$; $19.4\left(\underline{\mathrm{C}}\left(\mathrm{CH}_{3}\right)_{3}\right) \mathrm{ppm}$. Anal. Calcd. for $\mathrm{C}_{29} \mathrm{H}_{35} \mathrm{ClN}_{2} \mathrm{OSi}, \mathrm{M}=$ 490: C, 70.92; H, 7.14; N, 5.70. Found: C, 71.19; H, 7.28; N, 5.89 .

\section{1-Methyl-4-(tert-butyldiphenylsilyloxymethylindole)-3-car- baldehyde (11)}

A solution of phosphorous oxychloride $(0.27 \mathrm{~mL}, 2.91$ $\mathrm{mmol})$ in anhydrous dimethylformamide $(1.3 \mathrm{~mL})$ was stirred at $0{ }^{\circ} \mathrm{C}$ for 30 min under an argon atmosphere. A solution of compound 4b $(933 \mathrm{mg}, 2.34 \mathrm{mmol})$ in anhydrous dimethylformamide $(5 \mathrm{~mL})$ was added dropwise. The reaction mixture was stirred at room temperature for $30 \mathrm{~min}$ and cooled to $0{ }^{\circ} \mathrm{C}$. Water $(10 \mathrm{~mL})$ was added and $20 \%$ aqueous $\mathrm{NaOH}$ was added dropwise until $\mathrm{pH}=8$. The solution was heated at $60{ }^{\circ} \mathrm{C}$ for $30 \mathrm{~min}$. The precipitate obtained upon cooling was filtered and dissolved in chloroform. This solution was dried over anhydrous $\mathrm{Na}_{2} \mathrm{SO}_{4}$ and evaporated to yield aldehyde 11 (891 $\mathrm{mg}, 89 \%)$, as a yellow solid. Mp 92$95{ }^{\circ} \mathrm{C}$. IR $(\mathrm{NaCl})$ v: $1669.1(\mathrm{CO}) \mathrm{cm}^{-1} .{ }^{1} \mathrm{H}-\mathrm{NMR}\left(\mathrm{CDCl}_{3}\right.$, $250 \mathrm{MHz}$ ) $8: 9.97$ (s, 1H, CHO); 7.77 (s, 1H, H-2); 7.71-7.67 (m, 4H, H-2",6"); 7.47-7.25 (m, 9H, H-5,6,7,3",4",5"); 5.30 $(\mathrm{s}, 2 \mathrm{H}, \mathrm{H}-\alpha) ; 3.85\left(\mathrm{~s}, 3 \mathrm{H}, \mathrm{N}-\mathrm{CH}_{3}\right) ; 1.07\left(\mathrm{~s}, 9 \mathrm{H}, \mathrm{C}\left(\mathrm{C}_{3}\right)_{3}\right)$ ppm. ${ }^{13} \mathrm{C}-\mathrm{NMR}\left(\mathrm{CDCl}_{3}, 62.9 \mathrm{MHz}\right) \delta$ : 185.4 (CHO); 138.2 (C-7a); 137.8 (C-2); 135.7 (C-2",6”); 133.7 (C-1"); 134.9 (C-4); 129.8 (C-3",5”); 129.7 (C-4"); 123.6 (C-3a); 122.7 
(C-5); 120.7 (C-6); 118.9 (C-3); 109.2 (C-7); 65.7 (C- $\alpha$ ); $34.0\left(\mathrm{~N}-C H_{3}\right) ; 27.0\left(\mathrm{C}\left(\mathrm{CH}_{3}\right)_{3}\right) ; 19.5\left(\underline{\mathrm{C}}\left(\mathrm{CH}_{3}\right)_{3}\right) \mathrm{ppm}$. Anal. Calcd. for $\mathrm{C}_{27} \mathrm{H}_{29} \mathrm{NO}_{2} \mathrm{Si}, \mathrm{M}=428: \mathrm{C}, 75.84 ; \mathrm{H}, 6.84 ; \mathrm{N}$, 3.28. Found: C, 75.53; H, 6.91; N, 3.29.

\section{1-Methyl-4-hydroxymethylindole-3-carbaldehyde (12) Method A}

The same procedure described for the preparation of 11, starting from $7.825 \mathrm{~g}(15.94 \mathrm{mmol})$ of compound $\mathbf{1 0}$ and basifying the aqueous solution to $\mathrm{pH} 12$ prior to heating at $60{ }^{\circ} \mathrm{C}$, gave $7.337 \mathrm{~g}(80 \%)$ of compound $\mathbf{1 2}$, as a white solid. Mp $109-113{ }^{\circ} \mathrm{C}$. IR (NaCl) v: $3380.8(\mathrm{OH}) ; 1644.6(\mathrm{CO}) \mathrm{cm}^{-}$ 1. ${ }^{\mathrm{H}} \mathrm{H}-\mathrm{NMR}\left(\mathrm{CDCl}_{3}, 250 \mathrm{MHz}\right) \delta: 9.68(\mathrm{~s}, 1 \mathrm{H}, \mathrm{CHO}) ; 7.74(\mathrm{~s}$, $1 \mathrm{H}, \mathrm{H}-2)$; 7.36-7.19 (m, 3H, H-5,6,7); 4.87 (s, 2H, H- $\alpha$ ); $3.85\left(\mathrm{~s}, 3 \mathrm{H}, \mathrm{N}-\mathrm{CH}_{3}\right) ; 1.03(\mathrm{~s}, 1 \mathrm{H}, \mathrm{OH}) \mathrm{ppm} .{ }^{13} \mathrm{C}-\mathrm{NMR}$ $\left(\mathrm{CDCl}_{3}, 62.9 \mathrm{MHz}\right) \delta$ : $185.4(\mathrm{CHO}) ; 144.5(\mathrm{C}-2) ; 139.6(\mathrm{C}-$ 7a); 136.6 (C-4); 124.7 (C-5); 124.5 (C-6); 122.6 (C-3a); 118.6 (C-3); 110.1 (C-7); $65.0(\mathrm{C}-\alpha) ; 34.2\left(\mathrm{~N}^{\left.-\mathrm{CH}_{3}\right)} \mathrm{ppm}\right.$. Anal. Calcd. for $\mathrm{C}_{11} \mathrm{H}_{11} \mathrm{NO}_{2}, \mathrm{M}=189: \mathrm{C}, 69.83 ; \mathrm{H}, 5.86 ; \mathrm{N}$, 7.40. Found: C, 69.50; H, 6.01; N, 7.10.

\section{Method B}

To a solution of compound $\mathbf{1 1}(3.410 \mathrm{~g}, 7.97 \mathrm{mmol})$ in acetonitrile $(1 \mathrm{~mL})$, was added dropwise, at $0{ }^{\circ} \mathrm{C}$, a $48 \%$ aqueous solution of $\mathrm{HF}(0.14 \mathrm{~mL}, 7.97 \mathrm{mmol})$. The solution was stirred at room temperature for $24 \mathrm{~h}$. After this time, the reaction mixture was poured onto a saturated aqueous solution of sodium bicarbonate $(15 \mathrm{~mL})$ and extracted with chloroform $(3 \times 15 \mathrm{~mL})$. The combined extracts were dried over anhydrous $\mathrm{Na}_{2} \mathrm{SO}_{4}$ and evaporated. The residue was chromatographed on silica gel, eluting with a 2:1 petroleum etherethyl acetate mixture, yielding $2.626 \mathrm{~g}(77 \%)$ of compound 12, as a white solid.

\section{Diethyl 2-((4'-tert-butyldiphenylsilyloxy-methyl-1'-methyl-} 3'-indolyl)methylene)malonate (13)

To a solution of diethyl malonate $(0,17 \mathrm{~mL})$ in pyridine ( $2 \mathrm{~mL}$ ), under an argon atmosphere, was added compound $12(200 \mathrm{mg}, 0.47 \mathrm{mmol})$, piperidine $(0.28 \mathrm{~mL})$ and $4 \mathrm{~A}$ molecular sieves. The reacting mixture was heated at $180^{\circ} \mathrm{C}$ for $48 \mathrm{~h}$, cooled poured on acidic water $(10 \mathrm{~mL})$ and extracted with ethyl acetate $(3 \times 10 \mathrm{~mL})$. The combined extracts were dried over anhydrous $\mathrm{Na}_{2} \mathrm{SO}_{4}$ and evaporated. The residue was chromatographed on silica gel, eluting with 10:1 petroleum ether-ethyl acetate, yielding $133 \mathrm{mg}$ (37\%) of compound 13, as a yellow solid. $\mathrm{Mp} 105-106^{\circ} \mathrm{C}$. IR $(\mathrm{NaCl}) \mathrm{v}$ : 1718.8 (CO); $1608.9 ; 1242.8 ; 1201.4 \mathrm{~cm}^{-1} .{ }^{1} \mathrm{H}-\mathrm{NMR}\left(\mathrm{CDCl}_{3}\right.$, $250 \mathrm{MHz}) \delta$ : 8.47 (s, 1H, H- $\left.\alpha^{\prime}\right) ; 7.70-7.66$ (m, 4H, H-2", 6"); 7.60 (s, 1H, H-2'); 7.43-7.36 (m, 6H, H-3",4",5"); 7.23 (d, $\left.1 \mathrm{H}, J=7,5 \mathrm{~Hz}, \mathrm{H}-5^{\prime}\right) ; 7.12$ (t, $1 \mathrm{H}, J=7,5 \mathrm{~Hz}, \mathrm{H}-6$ '); 6.92 (d, $1 \mathrm{H}, J=7,5 \mathrm{~Hz}, \mathrm{H}-7$ '); 5.13 (s, 2H, H- $\alpha) ; 4.32$ (q, 2H, $J=$ $\left.7,5 \mathrm{~Hz}, \mathrm{C}_{2} \mathrm{CH}_{3}\right) ; 4.18$ (q, $\left.2 \mathrm{H}, J=7.5 \mathrm{~Hz}, \mathrm{C}_{2} \mathrm{CH}_{3}\right) ; 3.79$ (s, $\left.3 \mathrm{H}, \mathrm{N}-\mathrm{CH}_{3}\right) ; 1.31$ (t, $\left.3 \mathrm{H}, J=7.5 \mathrm{~Hz}, \mathrm{CH}_{2} \mathrm{CH}_{3}\right) ; 1.19$ (t, $\left.3 \mathrm{H}, J=7.5 \mathrm{~Hz}, \mathrm{CH}_{2} \mathrm{C}_{3}\right) ; 1.03\left(\mathrm{~s}, 9 \mathrm{H}, \mathrm{C}\left(\mathrm{CH}_{3}\right)_{3}\right) \mathrm{ppm}{ }^{13} \mathrm{C}-$ NMR $\left(\mathrm{CDCl}_{3}, 62.9 \mathrm{MHz}\right) \delta$ : $168.6\left(\mathrm{CO}_{2} \mathrm{Et}\right) ; 165.0\left(\mathrm{CO}_{2} \mathrm{Et}\right)$; 138.1 (C- $\left.\alpha^{\prime}\right)$; 137.5 (C-7a'); 135.9 (C-2",6"); 133.8 (C-1"); 133.5 (C-4'); 131.7 (C-2'); 129.7 (C-4"); 127.7 (C-3",5”); 125.7 (C-3a'); 122.5 (C-5'); 121.3 (C-6'); 120.5 (C-2); 110.2 (C-3'); 109.6 (C-7'); 65.2 (C- $\alpha) ; 61.5\left(\mathrm{CH}_{2} \mathrm{CH}_{3}\right) ; 60.9$ $\left(\underline{\mathrm{CH}}_{2} \mathrm{CH}_{3}\right) ; 33.7\left(\mathrm{~N}^{-\mathrm{CH}_{3}}\right) ; 26.9\left(\mathrm{C}\left(\underline{\mathrm{CH}}_{3}\right)_{3}\right) ; 19.4\left(\underline{\mathrm{C}}\left(\mathrm{CH}_{3}\right)_{3}\right)$; $14.4\left(\mathrm{CH}_{2} \mathrm{CH}_{3}\right) ; 14.2\left(\mathrm{CH}_{2} \mathrm{CH}_{3}\right)$ ppm. Anal. Calcd. for $\mathrm{C}_{34} \mathrm{H}_{39} \mathrm{NO}_{5} \mathrm{Si}, \mathrm{M}=569: \mathrm{C}, 71.67, \mathrm{H}, 6.90, \mathrm{~N}, 2.46$. Found: C, 71.69, H, 7.08, N, 2.31.
Diethyl 2-((4'-hydroxymethyl-1'-methyl-3'-indolyl)methylene)malonate (14)

\section{$\underline{\operatorname{Method} A}$}

The same procedure described for the preparation of $\mathbf{1 3}$, starting from $367 \mathrm{mg}$ (1.94 mmol) of compound 12, afforded $115 \mathrm{mg}(22 \%)$ of 14 , as a yellow solid. $\mathrm{Mp} 75^{\circ} \mathrm{C}$. IR $(\mathrm{NaCl})$ v: $3464.0(\mathrm{OH}) ; 1717.8(\mathrm{CO}) ; 1605.4 ; 1241.9 ; 1202.4 \mathrm{~cm}^{-1}$. ${ }^{1} \mathrm{H}-\mathrm{NMR}\left(\mathrm{CDCl}_{3}, 250 \mathrm{MHz}\right) \delta: 8.47$ (s, $\left.1 \mathrm{H}, \mathrm{H}-\alpha\right)$ ') 7.65 (s, 1H, H-2'); 7.31-7.21 (m, 3H, H-5',6', 7'); 5.05 (d, 2H, $J=5$ $\mathrm{Hz}, \mathrm{H}-\alpha)$; 4.36-4.23 (m, 4H, $\left.2 \mathrm{C}_{2} \mathrm{CH}_{3}\right) ; 3.80(\mathrm{~s}, 3 \mathrm{H}, \mathrm{N}-$ $\left.\mathrm{CH}_{3}\right) ; 1.34-1.22\left(\mathrm{~m}, 6 \mathrm{H}, 2 \mathrm{CH}_{2} \mathrm{CH}_{3}\right) ; 1.90(\mathrm{~s}, 1 \mathrm{H}, \mathrm{OH}) \mathrm{ppm}$. ${ }^{13} \mathrm{C}-\mathrm{NMR}\left(\mathrm{CDCl}_{3}, 62.9 \mathrm{MHz}\right) \delta: 168.3\left(\mathrm{CO}_{2} \mathrm{Et}\right) ; 165.5$ $\left(\mathrm{CO}_{2} \mathrm{Et}\right) ; 137.6\left(\mathrm{C}-\alpha^{\prime}\right) ; 137.6\left(\mathrm{C}^{\prime} 7 \mathrm{a}^{\prime}\right) ; 133.4\left(\mathrm{C}-4^{\prime}\right) ; 132.2$ (C-2'); 126.0 (C-3a'); 122.8 (C-5'); 122.0 (C-6'); 120.1 (C2); 110.2 (C-7'); 110.0 (C-3'); 64.2 (C- $\alpha)$; $61.5\left(\mathrm{CH}_{2} \mathrm{CH}_{3}\right)$; $61.2\left(\mathrm{CH}_{2} \mathrm{CH}_{3}\right) ; \quad 33.7\left(\mathrm{~N}-\mathrm{CH}_{3}\right) ; 26.9\left(\mathrm{C}\left(\mathrm{CH}_{3}\right)_{3}\right) ; 14.4$ $\left(\mathrm{CH}_{2} \mathrm{CH}_{3}\right) ; 14.2 \quad\left(\mathrm{CH}_{2} \mathrm{CH}_{3}\right)$ ppm. Anal. Calcd. for $\mathrm{C}_{18} \mathrm{H}_{21} \mathrm{NO}_{5}, \mathrm{M}=331: \mathrm{C}, 65.24 ; \mathrm{H}, 6.39 ; \mathrm{N}, 4.23$. Found: $\mathrm{C}$, 64.96; H, 6.25; N, 4.09 .

\section{Method B}

To a solution of compound $\mathbf{1 3}(60 \mathrm{mg}, 0.11 \mathrm{mmol})$ in dry dichloromethane $(2 \mathrm{~mL})$ was added a $1 \mathrm{M}$ solution of tetrabutylammonium fluoride in tetrahydrofuran $(0.37 \mathrm{~mL}$, $1.26 \mathrm{mmol})$. The reaction mixture was stirred at room temperature for $16 \mathrm{~h}$, poured onto $\mathrm{pH} 7$ aqueous buffer $(0.5$ $\mathrm{mL})$ and extracted with ethyl ether $(3 \times 10 \mathrm{~mL})$. The combined extracts were dried over anhydrous $\mathrm{Na}_{2} \mathrm{SO}_{4}$ and evaporated. The residue was chromatographed on silica gel, eluting with 2:1 petroleum ether-ethyl acetate, yielding 27 $\mathrm{mg}$ (77\%) of compound 14.

Ethyl 3-(4'-tert-butyldiphenylsilyloxy-methyl-1'-methyl-indole-3'-yl)-3-oxopropanoate (15)

To a solution of ethyl diazoacetate $(0.14 \mathrm{~mL}, 1.39 \mathrm{mmol})$ and $\mathrm{BF}_{3}-\mathrm{Et}_{2} \mathrm{O}$ complex $(20 \mu \mathrm{L}, 0.16 \mathrm{mmol})$ in dry dichloromethane $(3 \mathrm{~mL})$, cooled to $0{ }^{\circ} \mathrm{C}$ and under an argon atmosphere, was added a solution of aldehyde $12(210 \mathrm{mg}, 0.5$ $\mathrm{mmol})$. The reacting mixture was stirred at room temperature for $2.5 \mathrm{~h}$, poured onto brine $(10 \mathrm{~mL})$ and extracted with diethyl ether $(3 \times 10 \mathrm{~mL})$. The combined extracts were dried over anhydrous $\mathrm{Na}_{2} \mathrm{SO}_{4}$ and evaporated. The residue was chromatographed on silica gel, eluting with 10:1 petroleum ether-ethyl acetate, yielding compound $1563 \mathrm{mg}, \mathbf{2 5 \%}$ ) as a pale yellow oil, formed by a ca. 1:2 mixture of oxo (15a) and enol $(\mathbf{1 5 b})$ tautomers. IR $(\mathrm{NaCl})$ v: $1731,8(\mathrm{CO}) \mathrm{cm}^{-1}$. Anal. Calcd. for $\mathrm{C}_{31} \mathrm{H}_{35} \mathrm{NO}_{4} \mathrm{Si}, \mathrm{M}=514: \mathrm{C}, 72.48 ; \mathrm{H}, 6.87 ; \mathrm{N}$, 2.73. Found: $\mathrm{C}, 72.27 ; \mathrm{H}, 6.58 ; \mathrm{N}, 2.44$

${ }^{1} \mathrm{H}-\mathrm{NMR}$ for 15a $\left(\mathrm{CDCl}_{3}, 250 \mathrm{MHz}\right) \delta$ : $7.66-7.62(\mathrm{~m}$, 4H, H-2",6”); 7.40-7.22 (m, 10H, H-2',5',6',7',3”,4”,5”); $5.15(\mathrm{~d}, 1 \mathrm{H}, J=15.6 \mathrm{~Hz}, \mathrm{H}-\alpha) ; 4.85(\mathrm{~d}, 1 \mathrm{H}, J=15.6 \mathrm{~Hz}, \mathrm{H}-$ $\alpha)$; 4.23-4.17 (m, 2H, $\mathrm{C}_{2} \mathrm{CH}_{3}$ ); 3.74 (s, $\left.3 \mathrm{H}, \mathrm{N}-\mathrm{CH}_{3}\right) ; 3.07$ (d, $1 \mathrm{H}, J=15.5 \mathrm{~Hz}, \mathrm{H}-2) ; 2.90(\mathrm{~d}, 1 \mathrm{H}, J=15.5 \mathrm{~Hz}, \mathrm{H}-2)$; $1.11\left(\mathrm{~s}, 9 \mathrm{H}, \mathrm{C}\left(\mathrm{CH}_{3}\right)_{3}\right) ; 0.84\left(\mathrm{t}, 3 \mathrm{H}, J=7,5 \mathrm{~Hz}, \mathrm{CH}_{2} \mathrm{CH}_{3}\right)$ ppm. ${ }^{13} \mathrm{C}-\mathrm{NMR}$ for 15a $\left(\mathrm{CDCl}_{3}, 62.9 \mathrm{MHz}\right) \delta: 197.0$ ( $\left.\underline{\mathrm{CO}}\right)$; 173.2 ( $\left.\mathrm{CO}_{2} \mathrm{Et}\right) ; 137.0$ (C-7a'); 135.6 (C-2”,6”); 134.4 (C-1"); 133.6 (C-4'); 129.8 (C-4”); 129.7 (C-2'); 127.8 (C-3",5”); 124.5 (C-3a'); 122.0 (C-5'); 116.1 (C-6'); 108.1 (C-7'); 99.6 $\left(\mathrm{C}-3^{\prime}\right) ; 68.3(\mathrm{C}-\alpha) ; 61.2\left(\mathrm{CH}_{2} \mathrm{CH}_{3}\right), 39.6(\mathrm{C}-2) ; 33.0(\mathrm{~N}-$ $\left.\mathrm{CH}_{3}\right) ; 27.1\left(\mathrm{C}\left(\underline{\mathrm{CH}}_{3}\right)_{3}\right) ; 19.5\left(\underline{\mathrm{C}}\left(\mathrm{CH}_{3}\right)_{3}\right) ; 14.1\left(\mathrm{CH}_{2} \underline{\mathrm{CH}}_{3}\right)$ ppm. 
${ }^{1} \mathrm{H}-\mathrm{NMR}$ for 15b $\left(\mathrm{CDCl}_{3}, 250 \mathrm{MHz}\right) \delta: 12.95(\mathrm{~s}, 1 \mathrm{H}$, $\mathrm{OH})$; 7.80-7.66 (m, 1H, H-7'); 7.66-7.62 (m, 4H, H-2",6"); 7.40-7.22 (m, 8H, H-5',6',3", 4",5"); 6.79 (s, 2H, H-2' у H2); $5.15(\mathrm{~d}, 1 \mathrm{H}, J=15,6 \mathrm{~Hz}, \mathrm{H}-\alpha) ; 4,85(\mathrm{~d}, 1 \mathrm{H}, J=15.6 \mathrm{~Hz}$, $\mathrm{H}-\alpha)$; 4.23-4.17 (m, 3H, N-CH $)$; 4.05-3.90 (m, 2H, $\mathrm{C}_{2} \mathrm{CH}_{3}$ ); 1.27 (t, $\left.3 \mathrm{H}, J=7.5 \mathrm{~Hz}, \mathrm{CH}_{2} \underline{\mathrm{CH}}_{3}\right) ; 1.13$ (s, $9 \mathrm{H}$, $\left.\mathrm{C}\left(\mathrm{CH}_{3}\right)_{3}\right) \mathrm{ppm} .{ }^{13} \mathrm{C}-\mathrm{NMR}$ for 15b $\left(\mathrm{CDCl}_{3}, 62.9 \mathrm{MHz}\right) \delta$ : 169.3 ( $\underline{\mathrm{CO}}) ; 168.8$ ( $\left.\underline{\mathrm{CO}}_{2} \mathrm{Et}\right) ; 137.0$ (C-7a'); 135.6 (C-2",6"); 134.4 (C-1"); 133.8 (C-4'); 129.8 (C-4”); 129.7 (C-2'); 127.8 (C-3”,5”); 124.5 (C-3a'); 122.0 (C-5'); 116.1 (C-6'); 108.1 (C-7'); 107.0 (C-3'); 67.8 (C- $\alpha) ; 60.9\left(\mathrm{CH}_{2} \mathrm{CH}_{3}\right) ; 33.0$ $\left(\mathrm{N}-\mathrm{CH}_{3}\right) ; 27.0\left(\mathrm{C}\left(\mathrm{CH}_{3}\right)_{3}\right) ; 19.4\left(\underline{\mathrm{C}}\left(\mathrm{CH}_{3}\right)_{3}\right) ; 14.3\left(\mathrm{CH}_{2} \mathrm{CH}_{3}\right)$ ppm.

Ethyl 3-(4'-tert-butyldiphenylsilyloxy-methyl-1'-methyl-indol-3'-yl)-2-propenoate, $E$ and $Z$ isomers (16)

\section{Method A}

A solution of aldehyde $11(5.758 \mathrm{~g}, 0.013 \mathrm{~mol})$ and ethoxycarbonylmethylenetriphenylphosphorane $(10.52 \mathrm{~g}$, $0.03 \mathrm{~mol})$ in absolute ethanol $(20 \mathrm{~mL})$ was refluxed for $9 \mathrm{~h}$ under an inert atmosphere. After the addition of an additional amount of phosphorane (10.52 g, $0.03 \mathrm{~mol})$, reflux was maintained for $14 \mathrm{~h}$. The reaction mixture was then cooled and the solvent was evaporated. Water $(20 \mathrm{~mL})$ was added and the suspension was extracted with ethyl acetate $(3 \times 20$ $\mathrm{mL}$ ). The combined extracts were dried over anhydrous $\mathrm{Na}_{2} \mathrm{SO}_{4}$ and evaporated. The residue was identified as a 9:1 mixture of the $E$ and $Z$ isomers of compound 16. Chromatography on silica gel, eluting with a 3:1 petroleum ether-ethyl acetate mixture, afforded $4.975 \mathrm{~g}(77 \%)$ of $(E)-16$, as a white solid, and $0.646 \mathrm{~g} \mathrm{(10 \% )}$ of $(Z)-16$, as a pale yellow oil.

Data for $(E)-\mathbf{1 6}$ : $\mathrm{Mp} 110^{\circ} \mathrm{C}$. IR $(\mathrm{NaCl})$ v: $2930.9 ; 1698.1$ (CO); 1619.9; 1530.4; $1257.7 \mathrm{~cm}^{-1} .{ }^{1} \mathrm{H}-\mathrm{NMR}\left(\mathrm{CDCl}_{3}, 250\right.$ $\mathrm{MHz}) \delta: 8.31(\mathrm{~d}, 1 \mathrm{H}, J=15 \mathrm{~Hz}, \mathrm{H}-3) ; 7.72-7.68(\mathrm{~m}, 4 \mathrm{H}, \mathrm{H}-$ 2",6"); 7.48 (s, 1H, H-2'); 7.41-7.31 (m, 6H, H-3",4",5"); 7.24 (d, $1 \mathrm{H}, J=7.5 \mathrm{~Hz}, \mathrm{H}-5$ '); 7.13 (t, $1 \mathrm{H}, J=7.5 \mathrm{~Hz}, \mathrm{H}-$ 6'); $6.96\left(\mathrm{~d}, 1 \mathrm{H}, J=7.5 \mathrm{~Hz}, \mathrm{H}-7^{\prime}\right) ; 6.18(\mathrm{~d}, 1 \mathrm{H}, J=15.0 \mathrm{~Hz}$, $\mathrm{H}-2$ ); 5.12 (s, $2 \mathrm{H}, \mathrm{H}-\alpha$ ); 4.18 (q, $2 \mathrm{H}, J=7.5 \mathrm{~Hz}, \mathrm{CH}_{2} \mathrm{CH}_{3}$ ); $3.80\left(\mathrm{~s}, 3 \mathrm{H}, \mathrm{N}-\mathrm{CH}_{3}\right) ; 1.23$ (t, $\left.3 \mathrm{H}, J=7.5 \mathrm{~Hz}, \mathrm{CH}_{2} \mathrm{CH}_{3}\right) ; 1.04$ (s, 9H, C $\left.\left(\mathrm{CH}_{3}\right)_{3}\right) .{ }^{13} \mathrm{C}-\mathrm{NMR}\left(\mathrm{CDCl}_{3}, 62.9 \mathrm{MHz}\right) \delta: 167.5$ $\left(\underline{\mathrm{CO}}_{2} \mathrm{Et}\right) ; 139.6(\mathrm{C}-3) ; 137.7$ (C-7a'); 135.7 (C-2",6”); 133.6 (C-1”); 133.3 (C-4'); 129.5 (C-4”); 128.4 (C-2'); 127.6 (C3",5"); 124.7 (C-3a'); 122.0 (C-5'); 120.4 (C-6'); 113.4 (C2); 112.5 (C-3'); 109.3 (C-7'); 65.0 (C- $\alpha) ; 59.8\left(\mathrm{CH}_{2} \mathrm{CH}_{3}\right)$, $33.3 \quad\left(\mathrm{~N}-\mathrm{CH}_{3}\right) ; 26.8 \quad\left(\mathrm{C}\left(\underline{\mathrm{CH}}_{3}\right)_{3}\right) ; 19.2 \quad\left(\underline{\mathrm{C}}\left(\mathrm{CH}_{3}\right)_{3}\right) ; 14.4$ $\left(\mathrm{CH}_{2} \mathrm{CH}_{3}\right)$ ppm. Anal. Calcd. for $\mathrm{C}_{31} \mathrm{H}_{35} \mathrm{NO}_{3} \mathrm{Si}, \mathrm{M}=497: \mathrm{C}$, 74.81; H, 7.09; N, 2.81. Found: C, 74.45; H, 7.33; N, 2.54.

Data for (Z)-17: IR (NaCl) v: $1700.6(\mathrm{CO})$; 1619.6; $1111.4 ; 1258.8 \mathrm{~cm}^{-1} .{ }^{1} \mathrm{H}-\mathrm{NMR}\left(\mathrm{CDCl}_{3}, 250 \mathrm{MHz}\right) \delta: 8.76(\mathrm{~s}$, $\left.1 \mathrm{H}, \mathrm{H}-2^{\prime}\right) ; 7.75$ (d, 1H, $\left.J=12.5 \mathrm{~Hz}, \mathrm{H}-3\right) ; 7.72-7.67$ (m, 4H, H-2",6"); 7.45-7.32 (m, 6H, H-3",4",5"); 7.26 (d, 1H, $J=$ $\left.7.5 \mathrm{~Hz}, \mathrm{H}-5^{\prime}\right) ; 7.15$ (t, $1 \mathrm{H}, J=7.5 \mathrm{~Hz}, \mathrm{H}-6$ '); 7.01 (d, 1H, $J$ $\left.=6.5 \mathrm{~Hz}, \mathrm{H}-7^{\prime}\right) ; 5.65(\mathrm{~d}, 1 \mathrm{H}, J=12.5 \mathrm{~Hz}, \mathrm{H}-2) ; 5.03(\mathrm{~s}, 2 \mathrm{H}$, $\mathrm{H}-\alpha) ; 4.19$ (q, $\left.2 \mathrm{H}, J=7.5 \mathrm{~Hz}, \mathrm{CH}_{2} \mathrm{CH}_{3}\right) ; 3.83(\mathrm{~s}, 3 \mathrm{H}, \mathrm{N}-$ $\left.\mathrm{CH}_{3}\right) ; 1.31\left(\mathrm{t}, 3 \mathrm{H}, J=7.5 \mathrm{~Hz}, \mathrm{CH}_{2} \mathrm{CH}_{3}\right) ; 1.03$ (s, $9 \mathrm{H}$, $\left.\mathrm{C}\left(\mathrm{C}_{3}\right)_{3}\right) \quad$ ppm. ${ }^{13} \mathrm{C}-\mathrm{NMR} \quad\left(\mathrm{CDCl}_{3}, \quad 62.9 \mathrm{MHz}\right) \delta: 168.1$ $\left(\mathrm{CO}_{2} \mathrm{Et}\right) ; 139.3(\mathrm{C}-3) ; 138.0\left(\mathrm{C}-7 \mathrm{a}^{\prime}\right) ; 133.6\left(\mathrm{C}-4^{\prime}\right) ; 129.0(\mathrm{C}-$ 2'); 124.8 (C-3a'); 122.4 (C-5'); 121.1 (C-6'); 113.2 (C-2);
112.4 (C-3'); 110.1 (C-7'); 64.1 (C- $\alpha) ; 60.2\left(\mathrm{CH}_{2} \mathrm{CH}_{3}\right), 33.6$ $\left(\mathrm{N}-\mathrm{CH}_{3}\right) ; 14.6\left(\mathrm{CH}_{2} \mathrm{CH}_{3}\right)$. Anal. Calcd. for $\mathrm{C}_{31} \mathrm{H}_{35} \mathrm{NO}_{3} \mathrm{Si}, \mathrm{M}$ $=497: \mathrm{C}, 74.81 ; \mathrm{H}, 7.09 ; \mathrm{N}, 2.81$. Found: $\mathrm{C}, 74.41 ; \mathrm{H}, 7.41$; $\mathrm{N}, 2.43$.

\section{Method B}

A solution of iminium salt $10(170 \mathrm{mg}, 0.35 \mathrm{mmol})$ and ethoxycarbonylmethylenetriphenylphosphorane $(183 \mathrm{mg}$, $0.52 \mathrm{mmol})$ in 1,1,2-trichloroethane $(2 \mathrm{~mL})$ was refluxed for $12 \mathrm{~h}$ under an inert atmosphere. After the addition of an additional amount of ylide (10.52 g, $0.03 \mathrm{~mol})$, reflux conditions were maintained for 4 days. After this time, the reaction mixture was cooled and the solvent was evaporated. Water $(20 \mathrm{~mL})$ was added and the suspension was extracted with ethyl acetate $(3 \times 20 \mathrm{~mL})$. The combined extracts were dried over anhydrous $\mathrm{Na}_{2} \mathrm{SO}_{4}$ and evaporated. Silica gel chromatography of the residue, eluting with a 3:1 petroleum ether-ethyl acetate mixture, afforded $110 \mathrm{mg}(64 \%)$ of $(\boldsymbol{E})$ 16 and $24 \mathrm{mg}$ (14\%) of (Z)-16.

(E)-Ethyl 3-(4'-hydroxymethyl-1'-methyl-indol-3'-yl)-2-propenoate (17)

The same method described for the preparation of $\mathbf{5 a}$, starting from $405 \mathrm{mg}(2.14 \mathrm{mmol})$ of aldehyde 12, afforded $361 \mathrm{mg}(67 \%)$ of $(E)-\mathbf{1 7}$, as a pale yellow solid. Mp 120-125 ${ }^{\circ} \mathrm{C}$. IR ( $\left.\mathrm{NaCl}\right)$ v: $3422.6(\mathrm{OH}) ; 1685.8(\mathrm{CO}) ; 1616.0 ; 1177.1$ $\mathrm{cm}^{-1} .{ }^{1} \mathrm{H}-\mathrm{NMR}\left(\mathrm{CDCl}_{3}, 250 \mathrm{MHz}\right) \delta: 8.25(\mathrm{~d}, 1 \mathrm{H}, J=15.0$ Hz, H-3); 7.47 (s, 1H, H-2'); 7.29-7.19 (m, 3H, H-5', 6', 7'); $6.19(\mathrm{~d}, 1 \mathrm{H}, J=15 \mathrm{~Hz}, \mathrm{H}-2) ; 5.07$ (s, $2 \mathrm{H}, \mathrm{H}-\alpha) ; 4.23(\mathrm{q}, 2 \mathrm{H}$, $\left.J=7.5 \mathrm{~Hz}, \mathrm{CH}_{2} \mathrm{CH}_{3}\right) ; 3.79\left(\mathrm{~s}, 3 \mathrm{H}, \mathrm{N}-\mathrm{CH}_{3}\right) ; 2.30(\mathrm{~s}, 1 \mathrm{H}$, $\mathrm{OH}) ; 1.31\left(\mathrm{t}, 3 \mathrm{H}, J=7.5 \mathrm{~Hz}, \mathrm{CH}_{2} \mathrm{CH}_{3}\right)$ ppm. ${ }^{13} \mathrm{C}-\mathrm{NMR}$ $\left(\mathrm{CDCl}_{3}, 62.9 \mathrm{MHz}\right) \delta$ : $168.1\left(\mathrm{CO}_{2} \mathrm{Et}\right) ; 139.3(\mathrm{C}-3) ; 138.0(\mathrm{C}-$ 7a'); 133.6 (C-4'); 129.0 (C-2'); 124.8 (C-3a'); 122.4 (C-5'); 121.1 (C-6'); 113.2 (C-2); 112.4 (C-3'); 110.0 (C-7'); 64.1 $(\mathrm{C}-\alpha) ; 60.2\left(\mathrm{CH}_{2} \mathrm{CH}_{3}\right), 33.6\left(\mathrm{~N}-\mathrm{CH}_{3}\right) ; 14.6\left(\mathrm{CH}_{2} \mathrm{CH}_{3}\right)$. Anal. Calcd. for $\mathrm{C}_{15} \mathrm{H}_{17} \mathrm{NO}_{3}, \mathrm{M}=259: \mathrm{C}, 69.48 ; \mathrm{H}, 6.61 ; \mathrm{N}, 5.40$. Found: C, 69.24; H, 6.68; N, 5.34.

\section{Ethyl 3-(4'-tert-butyldiphenylsilyloxy-methyl-1'-methyl-in- dol-3'-yl)propanoate (18)}

To a solution of compound $16(1 \mathrm{~g}, 2.01 \mathrm{mmol})$ in methanol $(20 \mathrm{~mL})$ was added $10 \% \mathrm{Pd}-\mathrm{C}(60 \mathrm{mg})$. The suspension was stirred at room temperature under a hydrogen atmosphere for $8.5 \mathrm{~h}$ and filtered through celite, which was washed with dichloromethane. Evaporation of the solvent afforded $1.01 \mathrm{~g} \mathrm{(100 \% )} \mathrm{of} \mathrm{compound} \mathrm{18,} \mathrm{as} \mathrm{a} \mathrm{pale} \mathrm{yellow}$ oil. IR (NaCl) v: 1731,8 (CO); $1111.7 \mathrm{~cm}^{-1} .{ }^{1} \mathrm{H}-\mathrm{NMR}$ $\left(\mathrm{CDCl}_{3}, 250 \mathrm{MHz}\right)$ 8: 7.70-7.67 (m, 4H, H-2",6”); 7.40-7.34 (m, 6H, H-3",4",5"); 7.17-7.11 (m, 3H, H-5',6',7'); 6.79 (s, $1 \mathrm{H}, \mathrm{H}-2$ '); $5.11(\mathrm{~s}, 2 \mathrm{H}, \mathrm{H}-\alpha) ; 4.05(\mathrm{q}, 2 \mathrm{H}, J=7,5 \mathrm{~Hz}$, $\left.\mathrm{C}_{2} \mathrm{CH}_{3}\right) ; 3.70\left(\mathrm{~s}, 3 \mathrm{H}, \mathrm{N}-\mathrm{CH}_{3}\right) ; 3.12(\mathrm{t}, 2 \mathrm{H}, J=7.5 \mathrm{~Hz}, \mathrm{H}-$ 3); 2.51 (t, $2 \mathrm{H}, J=7.5 \mathrm{~Hz}, \mathrm{H}-2) ; 1.16$ (t, $3 \mathrm{H}, J=7.5 \mathrm{~Hz}$, $\left.\mathrm{CH}_{2} \mathrm{CH}_{3}\right) ; 1.05$ (s, $\left.9 \mathrm{H}, \mathrm{C}\left(\mathrm{CH}_{3}\right)_{3}\right)$ ppm. ${ }^{13} \mathrm{C}-\mathrm{NMR}\left(\mathrm{CDCl}_{3}\right.$, $62.9 \mathrm{MHz})$ 8: $173.3\left(\mathrm{CO}_{2} \mathrm{Et}\right) ; 137.6$ (C-7a'); 135.8 (C-2",6"); 133.8 (C-1"); 133.5 (C-4'); 129.7 (C-4"); 127.8 (C-3",5"); 126.8 (C-2'); 124.8 (C-3a'); 121.5 (C-5'); 118.1 (C-6'); 114.0 (C-3'); 108.7 (C-7'); 64.6 (C- $\alpha) ; 60.3\left(\mathrm{CH}_{2} \mathrm{CH}_{3}\right), 36.0$

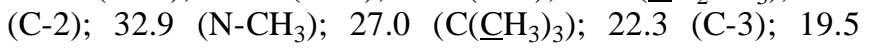
$\left(\underline{\mathrm{C}}\left(\mathrm{CH}_{3}\right)_{3}\right) ; 14.3 \quad\left(\mathrm{CH}_{2} \mathrm{CH}_{3}\right)$ ppm. Anal. Calcd. for $\mathrm{C}_{31} \mathrm{H}_{37} \mathrm{NO}_{3} \mathrm{Si}, \mathrm{M}=499: \mathrm{C}, 74.51 ; \mathrm{H}, 7.46 ; \mathrm{N}, 2.80$. Found: C, 74.25; H, 7.63; N, 2.74. 


\section{Ethyl 3-(4'-hydroxymethyl-1'-methyl-indol-3'-yl)propa- noate (19)}

\section{$\underline{\text { Method A }}$}

The same method described for the preparation of $\mathbf{1 8}$, starting from $200 \mathrm{mg}(0.77 \mathrm{mmol})$ of compound 17, afforded $192 \mathrm{mg}(95 \%)$ of 19 , as a pale yellow oil. IR $(\mathrm{NaCl}) \mathrm{v}$ : $3428,0(\mathrm{OH}) ; 1727.9(\mathrm{CO}) \mathrm{cm}^{-1} .{ }^{1} \mathrm{H}-\mathrm{NMR}\left(\mathrm{CDCl}_{3}, 250\right.$ MHz) $\delta: 7.23\left(\mathrm{~d}, 1 \mathrm{H}, J=7.5 \mathrm{~Hz}, \mathrm{H}-5{ }^{\prime}\right) ; 7.16(\mathrm{t}, 1 \mathrm{H}, J=7.5$ Hz, H-6'); 7.06 (d, 1H, J = 7.5 Hz, H-7'); 6.87 (s, 1H, H-2'); 5.01 (s, 2H, H- $\alpha$ ); 4.11 (q, $2 \mathrm{H}, J=7.5 \mathrm{~Hz}, \mathrm{CH}_{2} \mathrm{CH}_{3}$ ); 3.72 (s, $\left.3 \mathrm{H}, \mathrm{N}-\mathrm{CH}_{3}\right) ; 3.26$ (t, $\left.2 \mathrm{H}, J=7.5 \mathrm{~Hz}, \mathrm{H}-3\right) ; 2.70$ (t, $2 \mathrm{H}, J$ $=7.5 \mathrm{~Hz}, \mathrm{H}-2) ; 1.87(\mathrm{~s}, 1 \mathrm{H}, \mathrm{OH}) ; 1.21(\mathrm{t}, 3 \mathrm{H}, J=7.5 \mathrm{~Hz}$, $\left.\mathrm{CH}_{2} \mathrm{CH}_{3}\right)$ ppm. ${ }^{13} \mathrm{C}-\mathrm{NMR}\left(\mathrm{CDCl}_{3}, 62.9 \mathrm{MHz}\right) \delta: 173.6$ $\left(\mathrm{CO}_{2} \mathrm{Et}\right) ; 137.6\left(\mathrm{C}-7 \mathrm{a}^{\prime}\right) ; 133.2\left(\mathrm{C}^{\prime} 4^{\prime}\right) ; 127.3\left(\mathrm{C}-2^{\prime}\right) ; 125.0(\mathrm{C}-$ 3a'); 121.6 (C-5'); 119.8 (C-6'); 113.8 (C-3'); 109.6 (C-7'); $64.3(\mathrm{C}-\alpha) ; 60.5\left(\mathrm{CH}_{2} \mathrm{CH}_{3}\right) ; 35.9(\mathrm{C}-2) ; 32.9\left(\mathrm{~N}^{-} \mathrm{CH}_{3}\right) ; 22.0$ (C-3); $14.3\left(\mathrm{CH}_{2} \mathrm{CH}_{3}\right)$ ppm. Anal. Calcd. for $\mathrm{C}_{15} \mathrm{H}_{19} \mathrm{NO}_{3}, \mathrm{M}$ $=261: \mathrm{C}, 68.94 ; \mathrm{H}, 7.33 ; \mathrm{N}, 5.36$. Found: $\mathrm{C}, 68.51 ; \mathrm{H}, 7.32$; N, 5.19 .

\section{Method B}

To a solution of compound $18(891 \mathrm{mg}, 1.79 \mathrm{mmol})$ in dry dichloromethane $(6 \mathrm{~mL})$ was added a $1 \mathrm{M}$ solution of tetrabutylammonium fluoride in tetrahydrofuran $(6.22 \mathrm{~mL}$, $21 \mathrm{mmol}$ ). The solution was stirred at room temperature for $16 \mathrm{~h}$, poured onto $\mathrm{pH} 7$ aqueous buffer $(35 \mathrm{~mL})$ and extracted with ethyl ether $(3 \times 20 \mathrm{~mL})$. The combined extracts were dried over anhydrous $\mathrm{Na}_{2} \mathrm{SO}_{4}$ and evaporated. The residue was chromatographed on silica gel, eluting with a 2:1 petroleum ether-ethyl acetate mixture, to yield $446 \mathrm{mg}$ (95\%) of compound 19.

\section{Ethyl3-(4'-iodomethyl-1'-methylindol-3'-yl)propanoate (20)}

To a solution of compound $\mathbf{1 9}(100 \mathrm{mg}, 0.38 \mathrm{mmol})$ and oven-dried sodium iodide $(115 \mathrm{mg}, 0.77 \mathrm{mmol})$ in dry acetonitrile $(1 \mathrm{~mL})$, cooled to $-78{ }^{\circ} \mathrm{C}$ and kept under an argon atmosphere, was added trimethylsilyl chloride $(0.1 \mathrm{~mL}, 0.77$ mmol). The temperature was raised to $-40{ }^{\circ} \mathrm{C}$ and the reaction mixture was stirred at this temperature for $1.5 \mathrm{~h}$. Addition of a $10 \%$ sodium thiosulfate aqueous solution caused the precipitation of a iodide $\mathbf{2 0}$ (136 $\mathrm{mg}, 96 \%)$, as a yellow solid. Mp 68-70 ${ }^{\circ} \mathrm{C}$. IR (NaCl) v: $1730.6(\mathrm{CO}) \mathrm{cm}^{-1} .{ }^{1} \mathrm{H}-\mathrm{NMR}$ $\left(\mathrm{CDCl}_{3}, 250 \mathrm{MHz}\right)$ 8: 7.21-7.17 (m, 1H, H-5'); 7.12-7.05 (m, 2H, H-6', 7'); 6.88 (s, 1H, H-2'); 4.89 (s, 2H, H- $\alpha$ ); 4.14 (q, $\left.2 \mathrm{H}, J=7.5 \mathrm{~Hz}, \mathrm{CH}_{2} \mathrm{CH}_{3}\right) ; 3.70\left(\mathrm{~s}, 3 \mathrm{H}, \mathrm{N}-\mathrm{CH}_{3}\right) ; 3.41(\mathrm{t}, 2 \mathrm{H}$, $J=7.5 \mathrm{~Hz}, \mathrm{H}-3) ; 2.74(\mathrm{t}, 2 \mathrm{H}, J=7.5 \mathrm{~Hz}, \mathrm{H}-2) ; 1.24(\mathrm{t}, 3 \mathrm{H}, J$ $\left.=7.5 \mathrm{~Hz}, \mathrm{CH}_{2} \mathrm{CH}_{3}\right)$ ppm. ${ }^{13} \mathrm{C}-\mathrm{NMR}\left(\mathrm{CDCl}_{3}, 62.9 \mathrm{MHz}\right) \delta$ : $173.6\left(\mathrm{CO}_{2} \mathrm{Et}\right) ; 137.6$ (C-7a'); 133.1 (C-4'); 127.6 (C-2'); 124.8 (C-3a'); 121.6 (C-5'); 121.2 (C-6'); 113.8 (C-3'); 109.8 (C-7'); $60.4\left(\mathrm{CH}_{2} \mathrm{CH}_{3}\right), 35.8(\mathrm{C}-2) ; 32.9\left(\mathrm{~N}-\mathrm{CH}_{3}\right)$; $22.4(\mathrm{C}-3) ; 14.1\left(\mathrm{CH}_{2} \mathrm{CH}_{3}\right) ; 6.6(\mathrm{C}-\alpha) \mathrm{ppm}$. Anal. Calcd. for $\mathrm{C}_{15} \mathrm{H}_{18} \mathrm{INO}_{2}, \mathrm{M}=371: \mathrm{C}, 48.53 ; \mathrm{H}, 4.89 ; \mathrm{N}, 3.77$. Found: $\mathrm{C}$, $48.89 ; \mathrm{H}, 5.12 ; \mathrm{N}, 3.85$.

\section{Ethyl3-(4'-nitromethyl-1'-methylindol-3'-yl)propanoate (21)}

Sodium nitrite $(55 \mathrm{mg}, 0.8 \mathrm{mmol})$ and urea $(62 \mathrm{mg}, 1.04$ mmol) were dried in an oven at $80{ }^{\circ} \mathrm{C}$ for $14 \mathrm{~h}$. Both compounds were dissolved in dry dimethylformamide $(4 \mathrm{~mL})$, under an argon atmosphere. The solution was cooled to -78 ${ }^{\circ} \mathrm{C}$ and a solution of iodide 20 (128 mg, $\left.0.35 \mathrm{mmol}\right)$ in dry dimethylformamide $(0.75 \mathrm{~mL})$ was added dropwise. The bath temperature was left to raise to $-40{ }^{\circ} \mathrm{C}$ and the reaction mixture was stirred at this temperature for $2 \mathrm{~h}$ and then warmed to room temperature. Water $(5 \mathrm{~mL})$ was added and the reaction mixture was extracted with ethyl acetate $(3 \times 10$ $\mathrm{mL}$ ). The combined extracts were dried over anhydrous $\mathrm{Na}_{2} \mathrm{SO}_{4}$ and evaporated. The residue was chromatographed on silica gel, eluting with a 8:1 petroleum ether-ethyl acetate mixture, to yield $46 \mathrm{mg}(54 \%)$ of nitro derivative 21 , as a pale yellow oil, and $5 \mathrm{mg}(7 \%)$ of alcohol 19, from hydrolysis of the starting iodide. IR ( NaCl) v: $1729.9(\mathrm{CO}) ; 1552.9$ and $1373.3\left(\mathrm{NO}_{2}\right) .{ }^{1} \mathrm{H}-\mathrm{NMR}\left(\mathrm{CDCl}_{3}, 250 \mathrm{MHz}\right) \delta: 7.35(\mathrm{~d}$, $1 \mathrm{H}, J=7.5 \mathrm{~Hz}, \mathrm{H}-5$ '); $7.22\left(\mathrm{t}, 1 \mathrm{H}, J=7.5 \mathrm{~Hz}, \mathrm{H}-6^{\prime}\right) ; 7.12$ (d, $1 \mathrm{H}, J=7.5 \mathrm{~Hz}, \mathrm{H}^{-7}$ ') 6.93 (s, 1H, H-2'); 5.84 (s, 2H, H$\alpha) ; 4.12$ (q, $\left.2 \mathrm{H}, J=7.5 \mathrm{~Hz}, \mathrm{CH}_{2} \mathrm{CH}_{3}\right) ; 3.74\left(\mathrm{~s}, 3 \mathrm{H}, \mathrm{N}-\mathrm{CH}_{3}\right)$; 3.19 (t, $2 \mathrm{H}, J=7.5 \mathrm{~Hz}, \mathrm{H}-3) ; 2.67$ (t, $2 \mathrm{H}, J=7.5 \mathrm{~Hz}, \mathrm{H}-2)$; 1.22 (t, $3 \mathrm{H}, J=7.5 \mathrm{~Hz}, \mathrm{CH}_{2} \mathrm{CH}_{3}$ ) ppm. Anal. Calcd. for $\mathrm{C}_{15} \mathrm{H}_{18} \mathrm{~N}_{2} \mathrm{O}_{4}, \mathrm{M}=290: \mathrm{C}, 62.06 ; \mathrm{H}, 6.25 ; \mathrm{N}, 9.65$. Found: $\mathrm{C}$, 61.87; H, 5.97; N, 9.44.

\section{3-(4'-tert-Butyldiphenylsilyloxymethyl-1'-methylindol-3'-yl) propanol (23)}

A solution of compound $18(150 \mathrm{mg}, 0.3 \mathrm{mmol})$ in dry tetrahydrofuran $(1.5 \mathrm{~mL})$ was added dropwise to a suspension of lithium aluminium hydride $(50 \mathrm{mg}, 0.8 \mathrm{mmol})$ in the same solvent $(1 \mathrm{~mL})$, under an argon atmosphere and at 0 ${ }^{\circ} \mathrm{C}$. The suspension was stirred at room temperature for $16 \mathrm{~h}$. Excess lithium aluminium hydride was destroyed by addition of ethyl acetate followed by a few drops of water, maintaining initially the reaction mixture in a bath at $0{ }^{\circ} \mathrm{C}$ and then at room temperature for $30 \mathrm{~min}$. Solid sodium bicarbonate was then added until a white solid was formed, which was filtered and washed with ethyl acetate. The organic solvent was dried over anhydrous $\mathrm{Na}_{2} \mathrm{SO}_{4}$ and evaporated, yielding compound $23(120 \mathrm{mg}, 88 \%)$ as a colourless oil. IR $(\mathrm{NaCl}) \mathrm{v}$ : $3355.1(\mathrm{OH}) ; 1111.6 \mathrm{~cm}^{-1} .{ }^{1} \mathrm{H}-\mathrm{NMR}\left(\mathrm{CDCl}_{3}, 250 \mathrm{MHz}\right) \delta$ : 7.73-7.70 (m, 4H, H-2",6"); 7.42-7.32 (m, 6H, H-3",4",5"); 7.25-7.18 (m, 3H, H-5',6', 7'); 6.78 (s, 1H, H-2'); 5.14 (s, $2 \mathrm{H}, \mathrm{H}-\alpha) ; 3.71\left(\mathrm{~s}, 3 \mathrm{H}, \mathrm{N}-\mathrm{CH}_{3}\right) ; 3.51(\mathrm{t}, 2 \mathrm{H}, J=7.5 \mathrm{~Hz}, \mathrm{H}-$ 1); 2.77 (q, $2 \mathrm{H}, J=7.5 \mathrm{~Hz}, \mathrm{H}-3) ; 1.77-1.65$ (m, 2H, H-2); $1.24(\mathrm{~s}, 1 \mathrm{H}, \mathrm{OH}) ; 1.08\left(\mathrm{~s}, 9 \mathrm{H}, \mathrm{C}\left(\mathrm{CH}_{3}\right)_{3}\right) \mathrm{ppm} .{ }^{13} \mathrm{C}-\mathrm{NMR}$ $\left(\mathrm{CDCl}_{3}, 62.9 \mathrm{MHz}\right) \delta$ : 137.5 (C-7a'); 135.8 (C-2",6”); 133.8 (C-1"); 133.8 (C-4'); 129.7 (C-4”); 127.8 (C-3",5"); 126.7 (C-2'); 124.8 (C-3a'); 121.5 (C-5'); 117.3 (C-6'); 114.8 (C3'); 108.5 (C-7'); 64.4 (C- $\alpha$ ); 62.6 (C-3); 34.6 (C-2); 32.8 $\left(\mathrm{N}-\mathrm{CH}_{3}\right) ; 27.0\left(\mathrm{C}\left(\mathrm{CH}_{3}\right)_{3}\right) ; 23.1(\mathrm{C}-3) ; 19.5\left(\underline{\mathrm{C}}\left(\mathrm{CH}_{3}\right)_{3}\right) \mathrm{ppm}$. Anal. Calcd. for $\mathrm{C}_{29} \mathrm{H}_{35} \mathrm{NO}_{2} \mathrm{Si}, \mathrm{M}=457$ : C, 76.10; H, 7.71; N, 3.06. Found: C, 76.40; H, 7.43; N, 2.96.

\section{3-(4'-tert-Butyldiphenylsilyloxymethyl-1'-methylindol-3'- yl)propanal (24)}

To a solution of compound $\mathbf{2 3}$ (300 $\mathrm{mg}, 0.66 \mathrm{mmol})$ and $\mathrm{N}$-methylmorfoline $\mathrm{N}$-oxide (116 mg, $1 \mathrm{mmol})$ in dry acetonitrile $(4 \mathrm{~mL})$, under an argon atmosphere, was added activated powdered $4 \AA$ molecular sieves and tetrapropylammonium perruthenate (TPAP) $(24 \mathrm{mg}, 0.066 \mathrm{mmol})$. The suspension was stirred at room temperature for $3 \mathrm{~h}$ and filtered through a pad of celite, which was washed with dichloromethane. Evaporation of the solvent followed by silica gel chromatography, eluting with a 3:1 petroleum ether-ethyl acetate mixture, afforded compound $\mathbf{2 4}(216 \mathrm{mg}, 72 \%)$ as a pale green, viscous oil. IR ( NaCl) v: $1724.8(\mathrm{CO}) ; 1112.0$ $\mathrm{cm}^{-1} .{ }^{1} \mathrm{H}-\mathrm{NMR}\left(\mathrm{CDCl}_{3}, 250 \mathrm{MHz}\right) \delta: 7.71-7.67(\mathrm{~m}, 4 \mathrm{H}, \mathrm{H}-$ 
2",6"); 7.44-7.31 (m, 6H, H-3",4",5"); 7.21-7.14 (m, 3H, H5',6',7'); 6.74 (s, 1H, H-2'); 5.09 (s, 2H, H- $\alpha) ; 3.70$ (s, 3H, $\left.\mathrm{N}-\mathrm{CH}_{3}\right) ; 3.12(\mathrm{t}, 2 \mathrm{H}, J=7.5 \mathrm{~Hz}, \mathrm{H}-3) ; 2.60(\mathrm{t}, 2 \mathrm{H}, J=7.5$ $\mathrm{Hz}, \mathrm{H}-2) ; 1.06\left(\mathrm{~s}, 9 \mathrm{H}, \mathrm{C}\left(\mathrm{CH}_{3}\right)_{3}\right) \mathrm{ppm} .{ }^{13} \mathrm{C}-\mathrm{NMR}\left(\mathrm{CDCl}_{3}\right.$, $62.9 \mathrm{MHz}$ ) $\delta$ : 202.5 (CHO); 137.5 (C-7a'); 135.7 (C-2”,6”); 133.6 (C-1"); 133.3 (C-4'); 129.7 (C-4”); 127.7 (C-3",5”); 126.8 (C-2'); 124.6 (C-3a'); 121.5 (C-5'); 118.1 (C-6'); 113.4 (C-3'); 108.7 (C-7'); 64.5 (C- $\alpha) ; 44.8$ (C-2); 32.7 (N$\left.\mathrm{CH}_{3}\right) ; 26.9\left(\mathrm{C}\left(\underline{\mathrm{CH}}_{3}\right)_{3}\right) ; 19.3(\mathrm{C}-3) ; 19,03\left(\underline{\mathrm{C}}\left(\mathrm{CH}_{3}\right)_{3}\right)$ ppm. Anal. Calcd. for $\mathrm{C}_{29} \mathrm{H}_{33} \mathrm{NO}_{2} \mathrm{Si}, \mathrm{M}=455$ : C, 76.48; H, 7.25; N, 3.07. Found: C, 76.23; H, 7.13; N, 2.92.

\section{3-(4'-tert-Butyldiphenylsilyloxymethyl-1'-methylindol-3'- yl)propanal dimethyl acetal (25)}

To a solution of trimethylsilyl triflate $(10 \mu \mathrm{L}, 0.05 \mathrm{mmol})$ in dry dichloromethane $(1 \mathrm{~mL})$, at $-78^{\circ} \mathrm{C}$ and under an argon atmosphere, was added methoxytrimetylsilane $(61 \mu \mathrm{L}, 0.44$ mmol). After stirring for $5 \mathrm{~min}$ at the same temperature, a solution of compound 24 (100 $\mathrm{mg}, 0.22 \mathrm{mmol})$ in dry dichloromethane $(3 \mathrm{~mL})$ was added and stirring at $-78{ }^{\circ} \mathrm{C}$ was maintained for $3 \mathrm{~h}$. Pyridine $(0.1 \mathrm{~mL})$ was then added, and the reaction was left to warm to rom temperature, washed with a sodium bicarbonate saturated aqueous solution (15 $\mathrm{mL})$, which was extracted with ethyl ether $(3 \times 10 \mathrm{~mL})$. The combined organic extracts were dried over anhydrous $\mathrm{Na}_{2} \mathrm{SO}_{4}$ and evaporated, yielding $80 \mathrm{mg}(73 \%)$ of compound 25, as a colourless oil. ${ }^{1} \mathrm{H}-\mathrm{NMR}\left(\mathrm{CDCl}_{3}, 250 \mathrm{MHz}\right) \delta$ : $7.75-$ 7.71 (m, 4H, H-2",6”); 7.44-7.33 (m, 6H, H-3",4”,5”); 7.217.19 (m, 3H, H-5',6',7'); 6.79 (s, 1H, H-2'); 5.17 (s, 2H, H$\alpha) ; 4.31$ (t, $1 \mathrm{H}, J=7.5 \mathrm{~Hz}, \mathrm{H}-1) ; 3.73$ (s, 3H, N-CH $\left.{ }_{3}\right) ; 3.24$ $\left(\mathrm{s}, 6 \mathrm{H}, \mathrm{O}\left(\mathrm{CH}_{3}\right)_{2}\right) ; 2.83(\mathrm{t}, 2 \mathrm{H}, J=7.5 \mathrm{~Hz}, \mathrm{H}-3) ; 1.80(\mathrm{t}, 2 \mathrm{H}$, $J=7.5 \mathrm{~Hz}, \mathrm{H}-2)$; 1.09 (s, 9H, $\left.\mathrm{C}\left(\mathrm{CH}_{3}\right)_{3}\right)$ ppm. Anal. Calcd. for $\mathrm{C}_{31} \mathrm{H}_{39} \mathrm{NO}_{3} \mathrm{Si}, \mathrm{M}=502$ : $\mathrm{C}, 74.21 ; \mathrm{H}, 7.83 ; \mathrm{N}, 2.79$. Found: C, 74.01; H, 7.67; N, 2.56.

\section{3-(4'-Hydroxymethyl-1'-methylindol-3'-yl)propanal dimeth- yl acetal (26)}

To a solution of silyl ether $25(80 \mathrm{mg}, 0.016 \mathrm{mmol})$ in dry dichloromethane $(1 \mathrm{~mL})$ was added a commercially available $1 \mathrm{M}$ solution of tetrabutylammonium fluoride in tetrahydrofuran $(0.6 \mathrm{~mL}, 1.92 \mathrm{mmol})$. The reacting mixture was stirred at room temperature for $16 \mathrm{~h}$. After this time, it was poured onto $\mathrm{pH} 7$ aqueous buffer $(3 \mathrm{~mL})$ and extracted with diethyl ether $(3 \times 10 \mathrm{~mL})$. The combined organic layers were dried over anhydrous $\mathrm{Na}_{2} \mathrm{SO}_{4}$ and evaporated. The residue was chromatographed on silica gel, eluting with a 10:1 petroleum ether-ethyl acetate mixture, to give $27 \mathrm{mg}$ $(67 \%)$ of compound $\mathbf{2 6}$, as a colourless oil. ${ }^{1} \mathrm{H}-\mathrm{NMR}$ $\left(\mathrm{CDCl}_{3}, 250 \mathrm{MHz}\right) \delta: 7.27\left(\mathrm{~d}, 1 \mathrm{H}, J=7.5 \mathrm{~Hz}, \mathrm{H}-5^{\prime}\right) ; 7.19(\mathrm{t}$, $\left.1 \mathrm{H}, J=7.5 \mathrm{~Hz}, \mathrm{H}-6^{\prime}\right)$; 7.09 (d, 1H, $J=7.5 \mathrm{~Hz}, \mathrm{H}-7^{\prime}$ ); 6.88 (s, 1H, H-2'); 5.04 (s, 2H, H- $\alpha) ; 4.51$ (t, 1H, J = $7.5 \mathrm{~Hz}, \mathrm{H}-$ 1); $3.75\left(\mathrm{~s}, 3 \mathrm{H}, \mathrm{N}-\mathrm{CH}_{3}\right) ; 3.36\left(\mathrm{~s}, 6 \mathrm{H}, 2 \mathrm{OCH}_{3}\right) ; 3.00(\mathrm{t}, 2 \mathrm{H}, J$ $=7.5 \mathrm{~Hz}, \mathrm{H}-3)$; 2.06-1.98 (m, 3H, H-2, OH) ppm. Anal. Calcd. for $\mathrm{C}_{15} \mathrm{H}_{21} \mathrm{NO}_{3}, \mathrm{M}=263$ : C, 68.42; H, 8.04; N, 5.32. Found: C, 68.23; H, 7.91; N, 5.14.

\section{3-(4'-Trimethylsilyloxymethyl-1'-methyl-indol-3'-yl)propa- nal dimethyl acetal (27)}

Neat trimethylsilyl chloride $(1 \mathrm{~mL})$ and triethylamine (1 $\mathrm{mL}$ ) were thoroughly mixed and the resulting suspension was centrifuged to eliminate triethylamine hydrochloride generated due to the presence of traces of hydrochloric acid in the commercial TMS chloride. $60 \mu \mathrm{L}$ of the supernatant, containing about $0.22 \mathrm{mmol}$ of trimethylsilyl chloride, were added to a solution of compound $26(0,025 \mathrm{~g}, 0,11 \mathrm{mmol})$ and sodium iodide ( $33 \mathrm{mg}, 0.22 \mathrm{mmol})$ in dry acetonitrile (1 $\mathrm{mL}$ ), under an argon atmosphere at $-78{ }^{\circ} \mathrm{C}$. The temperature was risen to $-40{ }^{\circ} \mathrm{C}$ and the reaction mixture was stirred at this temperature for $1.5 \mathrm{~h}$. The reaction was warmed to room temperature, poured onto $10 \%$ aqueous sodium thiosulfate ( $3 \mathrm{~mL})$ and extracted with diethyl ether $(4 \times 5 \mathrm{~mL})$. The combined extracts were dried over anhydrous $\mathrm{Na}_{2} \mathrm{SO}_{4}$ and evaporated, yielding $25 \mathrm{mg}(75 \%)$ of compound 27 as a pale yellow oil. IR $(\mathrm{NaCl})$ v: $1250.4 ; 1072.1 \mathrm{~cm}^{-1}$. ${ }^{1} \mathrm{H}-\mathrm{NMR}$ $\left(\mathrm{CDCl}_{3}, 250 \mathrm{MHz}\right) \delta$ : 7.24-7.09 (m, 3H, H-5',6', '7'); 6.83 (s, $1 \mathrm{H}, \mathrm{H}-2$ '); 5.08 (s, 2H, H- $\alpha$ ); 4.50 (t, $1 \mathrm{H}, J=7.5 \mathrm{~Hz}, \mathrm{H}-1)$; $3.75\left(\mathrm{~s}, 3 \mathrm{H}, \mathrm{N}-\mathrm{CH}_{3}\right) ; 3.36\left(\mathrm{~s}, 6 \mathrm{H}, \mathrm{O}\left(\mathrm{CH}_{3}\right)_{2}\right) ; 2.96(\mathrm{t}, 2 \mathrm{H}, J=$ $7.5 \mathrm{~Hz}, \mathrm{H}-3) ; 2.02-1.96$ (m, 2H, H-2); 0.16 (s, 9H, Si $\left.\left(\mathrm{CH}_{3}\right)_{3}\right)$ ppm. ${ }^{13} \mathrm{C}-\mathrm{NMR}\left(\mathrm{CDCl}_{3}, 62.9 \mathrm{MHz}\right) \delta$ : $137.9\left(\mathrm{C}-7 \mathrm{a}^{\prime}\right) ; 133.8$ (C-4'); 126.9 (C-2'); 125.2 (C-3a'); 121.6 (C-5'); 118.6 (C6'); 115.0 (C-3'); 109.1 (C-7'); 104.3 (C-1); 63.7 (C- $\alpha$ ); 53.0 $\left(\mathrm{OCH}_{3}\right) ; 52.9\left(\mathrm{OCH}_{3}\right) ; 34.1(\mathrm{C}-2) ; 33.1\left(\mathrm{~N}-\mathrm{CH}_{3}\right) ; 22.2(\mathrm{C}-3)$; $0.0\left(\mathrm{Si}\left(\mathrm{CH}_{3}\right)_{3}\right)$ ppm. Anal. Calcd. for $\mathrm{C}_{18} \mathrm{H}_{29} \mathrm{NO}_{3} \mathrm{Si}, \mathrm{M}=335$ : C, 64.44; H, 8.71; N, 4.17. Found: C, 64.26; H, 8.54; N, 3.98 .

\section{4-(4'-tert-Butyldiphenylsilyloxymethyl-1'-methylindol-3'- yl)-2-butanone (28)}

To a solution of compound $\mathbf{4 b}(750 \mathrm{mg}, 1.9 \mathrm{mmol})$ in dry acetonitrile $(4 \mathrm{~mL})$ was added ytterbium (III) triflate $(50 \mathrm{mg}$, $0.081 \mathrm{mmol})$ and methyl vinyl ketone $(0.7 \mathrm{~mL}, 8.41 \mathrm{mmol})$. The solution was stirred at room temperature for $16 \mathrm{~h}$, under an argon atmosphere. The solvent was then evaporated and the residue was chromatographed on silica gel, eluting with 20:1 petroleum ether-diethyl ether, to give $1.153 \mathrm{~g} \mathrm{(74 \% )}$ of compound 28. IR $(\mathrm{NaCl}) \mathrm{v}$ : $1715.9(\mathrm{CO}) ; 1111.5 \mathrm{~cm}^{-1} .{ }^{1} \mathrm{H}-$ NMR $\left(\mathrm{CDCl}_{3}, 250 \mathrm{MHz}\right) \delta$ : 7.74-7.70 (m, 4H, H-2",6”); 7.44-7.34 (m, 6H, H-3",4",5"); 7.22-7.17 (m, 3H, H5',6',7'); 6.79 (s, 1H, H-2'); 5.13 (s, 2H, H- $\alpha) ; 3.72$ (s, 3H, $\left.\mathrm{N}-\mathrm{CH}_{3}\right) ; 3.04(\mathrm{t}, 2 \mathrm{H}, J=7.5 \mathrm{~Hz}, \mathrm{H}-4) ; 2.59(\mathrm{t}, 2 \mathrm{H}, J=7.5$ $\mathrm{Hz}, \mathrm{H}-3) ; 1.98$ (s, 3H, H-1); 1.09 (s, 9H, C( $\left.\left.\mathrm{CH}_{3}\right)_{3}\right)$ ppm. ${ }^{13} \mathrm{C}-$ NMR $\left(\mathrm{CDCl}_{3}, 62.9 \mathrm{MHz}\right) \delta: 209.3$ (CO); 136.1 (C-7a'); 136.0 (C-2",6”); 134.0 (C-1"); 133.7 (C-4'); 130.0 (C-4”); 128.1 (C-3",5”); 127.3 (C-2'); 125.0 (C-3a'); 121.8 (C-5'); 118.0 (C-6'); 113.4 (C-3'); 108.9 (C-7'); 64.7 (C- $\alpha)$; 45.7 (C-3); $33.1\left(\mathrm{~N}-\mathrm{CH}_{3}\right) ; 30.2(\mathrm{C}-1) ; 27.3\left(\mathrm{C}\left(\mathrm{CH}_{3}\right)_{3}\right) ; 21.3(\mathrm{C}-4)$; $19.7\left(\underline{\mathrm{C}}\left(\mathrm{CH}_{3}\right)_{3}\right)$ ppm. Anal. Calcd. for $\mathrm{C}_{30} \mathrm{H}_{35} \mathrm{NO}_{2} \mathrm{Si}, \mathrm{M}=$ 470: C, 76.71; H, 7.51; N, 2.98. Found: C, 76.42; H, 7.32; N, 2.72 .

\section{4-(4'-Hydroxymethyl-1'-methylindol-3'-yl)-2-butanone (29)}

To a solution of compound $28(621 \mathrm{mg}, 1.32 \mathrm{mmol})$ in dry dichloromethane $(4 \mathrm{~mL})$, was added a $1 \mathrm{M}$ solution of tetrabutylammonium fluoride in tetrahydrofuran $(4.6 \mathrm{~mL}, 15$ mmol). The solution was stirred at room temperature for 16 $\mathrm{h}$, poured onto $\mathrm{pH} 7$ aqueous buffer $(24 \mathrm{~mL})$ and extracted with dichloromethane ( 3 x $15 \mathrm{~mL})$. The combined organic layers were dried over anhydrous $\mathrm{Na}_{2} \mathrm{SO}_{4}$ and evaporated. The residue was chromatographed on silica gel, eluting with a 10:1 petroleum ether-ethyl acetate mixture, to yield $155 \mathrm{mg}$ (51\%) of compound 29, as a pale yellow solid. Mp $93{ }^{\circ} \mathrm{C}$. IR $(\mathrm{NaCl})$ v: $3388.1(\mathrm{OH}) ; 1702.2(\mathrm{CO}) ; 747.1 \mathrm{~cm}^{-1} .{ }^{1} \mathrm{H}-\mathrm{NMR}$ $\left(\mathrm{CDCl}_{3}, 250 \mathrm{MHz}\right) \delta: 7.27(\mathrm{~d}, 1 \mathrm{H}, J=7.5 \mathrm{~Hz}, \mathrm{H}-5$ '); $7.19(\mathrm{t}$, $1 \mathrm{H}, J=7.5 \mathrm{~Hz}, \mathrm{H}-6$ ' ); 7.08 (d, 1H, $J=7.5 \mathrm{~Hz}, \mathrm{H}-7^{\prime}$ ); 6.87 (s, 1H, H-2'); 5.03 (s, 2H, H- $\alpha$ ); 3.74 (s, 3H, N-CH ${ }_{3}$ ); 3.24 $(\mathrm{t}, 2 \mathrm{H}, J=7.5 \mathrm{~Hz}, \mathrm{H}-4) ; 2.87(\mathrm{t}, 2 \mathrm{H}, J=7.5 \mathrm{~Hz}, \mathrm{H}-3) ; 2.16$ 
(s, 3H, H-1); 1.84 (s, $1 \mathrm{H}, \mathrm{OH})$ ppm. ${ }^{13} \mathrm{C}-\mathrm{NMR}\left(\mathrm{CDCl}_{3}, 62.9\right.$ MHz) $\delta$ : 209.4 (CO); 138.1 (C-7a'); 133.4 (C-4'); 127.7 (C2'); 125.0 (C-3a'); 121.8 (C-5'); 120.1 (C-6'); 114.3 (C-3'); 110.0 (C-7'); $64.6(\mathrm{C}-\alpha)$; $45.4(\mathrm{C}-3) ; 33.2\left({\left.\mathrm{~N}-\mathrm{CH}_{3}\right)}^{2}\right) 30.4(\mathrm{C}-$ 1); 21.0 (C-4); $19.7\left(\underline{\mathrm{C}}\left(\mathrm{CH}_{3}\right)_{3}\right)$ ppm. Anal. Calcd. for $\mathrm{C}_{14} \mathrm{H}_{17} \mathrm{NO}_{2}, \mathrm{M}=231: \mathrm{C}, 72.70 ; \mathrm{H}, 7.41 ; \mathrm{N}, 6.06$. Found: $\mathrm{C}$, 72.35; H, 7.30; N, 5.90.

\section{4-(4'-Iodomethyl-1'-methylindol-3'-yl)-2-butanone (30)}

To a solution of compound 29 (100 $\mathrm{mg}, 0.43 \mathrm{mmol})$ and sodium iodide $(130 \mathrm{mg}, 0.86 \mathrm{mmol})$ in dry acetonitrile (2 $\mathrm{mL}$ ), kept at $-78{ }^{\circ} \mathrm{C}$ and under an argon atmosphere, was added trimethylsilyl chloride $(0.11 \mathrm{~mL}, 0.86 \mathrm{mmol})$. The temperature was risen to $-40{ }^{\circ} \mathrm{C}$ and the reaction mixture was stirred at this temperature for $1.5 \mathrm{~h}$. The reaction was warmed to room temperature and was poured onto $10 \%$ aqueous sodium thiosulfate $(3 \mathrm{~mL})$. The yellow precipitate (110 mg, $75 \%$ ) was identified as compound 30. Mp: $70{ }^{\circ} \mathrm{C}$. IR ( $\mathrm{NaCl})$ v: $1710,8(\mathrm{CO}) \mathrm{cm}^{-1} .{ }^{1} \mathrm{H}-\mathrm{NMR}\left(\mathrm{CDCl}_{3}, 250 \mathrm{MHz}\right)$

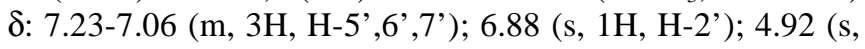
$2 \mathrm{H}, \mathrm{H}-\alpha) ; 3.72$ (s, $\left.3 \mathrm{H}, \mathrm{N}-\mathrm{CH}_{3}\right) ; 3.35$ (t, $2 \mathrm{H}, J=7.5 \mathrm{~Hz}, \mathrm{H}-$ 4); $2.92(\mathrm{t}, 2 \mathrm{H}, J=7.5 \mathrm{~Hz}, \mathrm{H}-3) ; 2.21(\mathrm{~s}, 3 \mathrm{H}, \mathrm{H}-1) \mathrm{ppm} .{ }^{13} \mathrm{C}-$ NMR $\left(\mathrm{CDCl}_{3}, 62.9 \mathrm{MHz}\right) \delta$ : 208.0 (CO); 138.1 (C-7a'); 133.4 (C-4'); 127.8 (C-2'); 125.0 (C-3a'); 121.6 (C-5'); 121.2 (C-6'); 113.5 (C-3'); 109.7 (C-7'); 45.0 (C-3); 32.7 $\left(\mathrm{N}-\mathrm{CH}_{3}\right) ; 29.8(\mathrm{C}-1) ; 21.1(\mathrm{C}-4) ; 19.7\left(\underline{\mathrm{C}}\left(\mathrm{CH}_{3}\right)_{3}\right) ; 6.7(\mathrm{C}-\alpha)$ ppm. Anal. Calcd. for $\mathrm{C}_{14} \mathrm{H}_{16} \mathrm{INO}, \mathrm{M}=341$ : C, 49.28, H, 4.73; N, 4.11. Found: C, 48.91; H, 4.72; N, 4.08.

\section{4-(4'-Nitromethyl-1'-methylindol-3'-yl)-2-butanone (31)}

Sodium nitrite $(50 \mathrm{mg}, 0.7 \mathrm{mmol})$ and urea $(55 \mathrm{mg}, 0.9$ mmol) were dried in an oven at $80{ }^{\circ} \mathrm{C}$ for $14 \mathrm{~h}$. These compounds were dissolved in dry dimethylformamide $(3 \mathrm{~mL})$, under an argon atmosphere. The solution was cooled to -78 ${ }^{\circ} \mathrm{C}$ and a solution of iodide $30(100 \mathrm{mg}, 0.30 \mathrm{mmol})$ in dry dimethylformamide $(1 \mathrm{~mL})$. The bath temperature was raised to $-40{ }^{\circ} \mathrm{C}$ and the reaction was stirred at this temperature for $2.5 \mathrm{~h}$ and extracted with dichloromethane $(3 \times 10 \mathrm{~mL})$. The combined extracts were dried over anhydrous $\mathrm{Na}_{2} \mathrm{SO}_{4}$ and evaporated. The residue was chromatographed on silica gel, eluting with a 5:1 petroleum ether-ethyl acetate mixture, to yield $12 \mathrm{mg}$ (35\%) of hydroxy ketone 29 and $12 \mathrm{mg}$ (31\%) of compound 31. ${ }^{1} \mathrm{H}-\mathrm{NMR}\left(\mathrm{CDCl}_{3}, 250 \mathrm{MHz}\right) \delta$ : $7.38(\mathrm{~d}$, $\left.1 \mathrm{H}, J=7.5 \mathrm{~Hz}, \mathrm{H}-5^{\prime}\right) ; 7.23$ (t, $\left.1 \mathrm{H}, J=5.0 \mathrm{~Hz}, \mathrm{H}-6^{\prime}\right) ; 7.15$ (d, $\left.1 \mathrm{H}, J=7.5 \mathrm{~Hz}, \mathrm{H}-5^{\prime}\right) ; 6.84$ (s, 1H, H-2'); 5.87 (s, $2 \mathrm{H}, \mathrm{H}-$ $\alpha) ; 3.76\left(\mathrm{~s}, 3 \mathrm{H}, \mathrm{N}-\mathrm{CH}_{3}\right) ; 3.17$ (t, $\left.2 \mathrm{H}, J=7.5 \mathrm{~Hz}, \mathrm{H}-4\right) ; 2.83$ (t, $2 \mathrm{H}, J=7.5 \mathrm{~Hz}, \mathrm{H}-3$ ); 2.17 (s, 3H, H-1) ppm. Anal. Calcd. for $\mathrm{C}_{14} \mathrm{H}_{16} \mathrm{~N}_{2} \mathrm{O}_{3}, \mathrm{M}=260$ : C, 64.60; H, 6.20; N, 10.76 . Found: C, 64.21; H, 5.93; N, 10.24.

\section{REFERENCES}

[1] Schardl, C. L.; Panaccione, D. G.; Tudzynski, P. Alkaloids Chem. Biol., 2006, 63, 45.

[2] Tsuda, M.; Mugishima, T.; Komatsu, K.; Sone, T.; Tanaka, M.; Mikami, Y.; Shiro, M.; Hirai, M.; Ohizumie, Y.; Kobayashi, J. Tetrahedron, 2003, 59, 3227.

[3] Menéndez, J. C. Top. Heterocycl. Chem., 2007, 11, in press.

[4] Stratmann, K.; Moore, R. E.; Bonjouklian, R.; Deeter, J. B.; Patterson, G. M. L.; Shaffer, S.; Smith, C. D.; Smitka, T. A. J. Am. Chem. Soc., 1994, 116, 9935.

[5] Sundberg, R. J. Indoles. Academic Press, 1996

[6] Gribble, G. W. J. Chem. Soc. Perkin Trans. 1, 2000, 1045.

[7] Tois, T.; Franzén, R.; Koskinen, A. Tetrahedron, 2003, 59, 5395.

[8] Barluenga, J.; Fañanás, F. J.; Sanz, R.; Fernández, R. Chem. Eur. J., 2002, 8, 2034.

[9] Clark, R. D.; Repke, D. B. Heterocycles, 1994, 22, 195.

[10] Somei, M.; Karasawa, Y.; Kaneko, C. Heterocycles, 1981, 16, 941.

[11] Holmquist, C. R.; Roskamp, E. J. Org. Chem., 1989, 54, 3258.

[12] Ballini, R.; Petrini, M.; Rosini, G. Synthesis, 1986, 849.

[13] Gelbard, G.; Colonna, S. Synthesis, 1977, 113.

[14] Kornblum, N.; Larson, H. O.; Blackwood, R. K.; Mooberry, D. D.; Oliveto, E. P.; Graham, G. E. J. Am. Chem. Soc., 1956, 78, 1497.

[15] Olah, G. A.; Narang, S. C.; Balaram Gupta, B. G.; Malhotra, R. J. Org. Chem., 1979, 44, 1247.

[16] Olah, G. A.; Balaram Gupta, B. G.; Malhotra, R.; Narang, S. C. J. Org. Chem., 1980, 45, 1638.

[17] Ono, N.; Kamimura, A.; Kaji, A. Synthesis, 1984, 226.

[18] Bergbreiter, D. E.; Lalonde, J. L. J. Org. Chem., 1987, 52, 1601.

[19] Seebach, D.; Lehr, F. Angew. Chem. Int. Ed. Engl., 1976, 15, 505.

[20] Shimizu, T.; Osako, K.; Nakata, T. Tetrahedron Lett., 1997, 38, 2685.

[21] Ley, S. V.; Norman, J.; Griffith, W. P.; Marsden, S. P. Synthesis, 1994, 639.

[22] Tsunoda, T.; Suzuki, M.; Noyori, R. Tetrahedron Lett., 1980, 21, 1357.

[23] Harrington, P. E.; Kerr, M. A. Synlett, 1996, 1047.

[24] Lin, W. W.; Jang, Y. J.; Wang, Y.; Liu, J. T.; Hu, S. R.; Wang, L. Y.; Yao, C. F. J. Org. Chem., 2001, 66, 1984. 